\title{
Analysis and validation of a new extended method for estimating plasma free cortisol including neutrophil elastase and competition from other steroids
}

\author{
Gudmand-Høyer, Johanne; Ottesen, Johnny T.
}

Published in:

The Journal of Steroid Biochemistry and Molecular Biology

DOI:

10.1016/j.jsbmb.2018.04.003

Publication date:

2018

Document Version

Early version, also known as pre-print

Citation for published version (APA):

Gudmand-Høyer, J., \& Ottesen, J. T. (2018). Analysis and validation of a new extended method for estimating plasma free cortisol including neutrophil elastase and competition from other steroids. The Journal of Steroid Biochemistry and Molecular Biology, 181, 109-124. https://doi.org/10.1016/j.jsbmb.2018.04.003

\section{General rights}

Copyright and moral rights for the publications made accessible in the public portal are retained by the authors and/or other copyright owners and it is a condition of accessing publications that users recognise and abide by the legal requirements associated with these rights.

- Users may download and print one copy of any publication from the public portal for the purpose of private study or research.

- You may not further distribute the material or use it for any profit-making activity or commercial gain.

- You may freely distribute the URL identifying the publication in the public portal.

\section{Take down policy}

If you believe that this document breaches copyright please contact rucforsk@kb.dk providing details, and we will remove access to the work immediately and investigate your claim. 


\title{
Analysis and validation of a new extended method for estimating plasma free cortisol including neutrophil elastase and competition from other steroids
}

\author{
Johanne Gudmand-Hoeyer ${ }^{a}$ and Johnny T. Ottesen ${ }^{a, *}$ \\ a Department of Science and Environment, Roskilde University, \\ * Corresponding author. E-mail address: johnny@ruc.dk, Postal address: \\ Department of Science and Environment, Roskilde University, Building 27.1, \\ 4000 Roskilde, Denmark
}

\begin{abstract}
A non-linear mechanistic model for the distribution of cortisol in plasma on free and bound forms is proposed. The influence of progesterone, testosterone and neutrophil elastase on the cortisol distribution in the blood is investigated.

The activity of neutrophil elastase is directly included in the model with the concentration of elastase and the kinetic constants describing the activity of elastase collected in one single input variable. The model is very sensitive towards this input variable and fits data excellently, when it is allowed to be subject specific.

The analysis shows that steroids such as testosterone with low affinity for corticosteroid-binding globulin $(\mathrm{CBG})$ do not significantly influence the concentration of free cortisol. Progesterone has a high affinity for CBG, but low plasma concentrations compared to cortisol. Contrary to expectations, progesterone is shown to impact the distribution of cortisol in plasma both under circumstances with high levels as seen in pregnancy and during the normal menstrual cycle of women.

Comparing the predictions of our model with predictions made with the equilibrium models by Coolens et al. [1], Dorin et al. [2] and Nguyen et al. [3] shows that the models differ considerably not only in their predictions for free cortisol, but also for cortisol on bound forms; i.e. bound to albumin, intact CBG and elastase-cleaved CBG.

Disregarding some of the smallest terms of the model equations a reduced version of the model in form of a fourth order polynomial equation is
\end{abstract}


obtained. The reduced version of the model performs almost identically to the full version and serves as a new formula for calculating the plasma free cortisol concentration.

Keywords:

Cortisol, Corticosteroid-Binding Globulin, Mechanism based model, Progesterone, Testosterone, Neutrophil elastase

\section{Introduction}

The steroid hormone cortisol is found in blood bound to the transport proteins corticosteroid-binding globulin (CBG), albumin (Alb), and in the free form with a distribution of $70 \%, 20 \%$ and $10 \%$, respectively [4]. Although free and bound cortisol contribute to the total concentration of cortisol in blood, only free cortisol is considered bioactive [5, 4]. By equilibrium dialysis, gel filtration or ultrafiltration direct measurement of free cortisol in human plasma can be done. However, the methods are both time-consuming and labour-intensive [6]. Thus, in most clinical procedures for measuring cortisol levels only the total cortisol concentration is measured [4]. Afterwards conservation and equilibrium assumptions are used to calculate concentrations of free and bound cortisol $[3,2]$.

The most often used formula for calculating free cortisol is the Coolens formula, which includes solving a second order polynomial [1, 2]. Coolens et al. [1] considered cortisol, CBG and albumin only and assumed the relevant reactions to be in equilibrium as well as conservation of the corresponding substances. Furthermore, the ratio of total albumin to its affinity for cortisol was assumed constant. Later Dorin et al. [2] developed an improved, cubic model with total albumin and its affinity for cortisol included as input variable and parameter, respectively, and by this excellent work demonstrated the importance of albumin concentration in cases with combined albumin and CBG deficiencies [2]. Nguyen et al. [3] extended the model further by considering two states of CBG: high-affinity, native $\mathrm{CBG}$ and low-affinity, elastase-cleaved $\mathrm{CBG}\left(\mathrm{CBG}^{*}\right)$, assuming equilibrium of the relevant reactions and conservation of the total amounts of the corresponding substances. This was elegantly generalized to a fourth order formula [3]. In their formula Nguyen et al. [3] ignored the actual enzymatic reaction cleaving CBG into $\mathrm{CBG}^{*}$ and instead took the total amounts of each of $\mathrm{CBG}$ and $\mathrm{CBG}^{*}$ to be conserved. 
Despite a high affinity for progesterone and a relatively high affinity for testosterone [7], the binding of progesterone and testosterone to CBG is often disregarded [1, 2, 3]. However, the concentrations of these two hormones varies considerably under both normal physiological and patophysiological circumstances. There are concentration differences between women and men $[8,9]$ and for women during the menstrual cycles $[10,11]$ and pregnancy $[12,13]$. For many women with polycystic ovaries or hirsutism increased levels of testosterone are observed [14].

In this paper we expand on the equilibrium considerations of cortisol's distribution in the blood by including testosterone and progesterone competing with cortisol in binding with CBG and albumin. In contrast to earlier work $[1,2,3]$, we include the enzymatic elastase reaction transforming native CBG into $\mathrm{CBG}^{*}$. The resulting equilibrium model can with small reductions be stated as a fourth order polynomial, which may serve as a new formula for calculating free cortisol.

The goal of this paper is to 1) make an improved formula for calculating free cortisol, 2) quantify the amount of cortisol binding to proteins in the bloodstream competing with other steroid hormones, 3) investigate the influence of neutrophil elastase, 4) compare the predictions made by the proposed models to prior models by Coolens et al. [1], Dorin et al. [2], Nguyen et al. [3] under separate physiologically relevant circumstances, and 5) investigate and discuss the impact of variation in parameter and input variable values resulting from an intensive literature study.

\section{Methods}

\subsection{Model development}

\subsubsection{The bio-chemistry of plasma cortisol concentration}

In this section we sketch the reactions of the bio-chemical system controlling cortisol's dynamic in the blood and name the variables included in the mathematical description in the following section 2.1.2. The chemical reactions are written schematically in box 2.1.1.

Let $X_{F}$ denote the concentration of free cortisol (Cort) in blood, $X_{C}$ that of free native $\mathrm{CBG}, X_{F C}$ that of cortisol bound to native CBG (Cort:CBG), $X_{A}$ the free albumin concentration in blood, and $X_{F A}$ that of cortisol bound to albumin (Cort:Alb).

During inflammation neutrophils may raise the level of neutrophil elastase (NE) in the blood. Elastase acts as an enzyme in transforming high-affinity, 
native $\mathrm{CBG}$ into a state $\mathrm{CBG}^{*}$ with lower affinity $[15,16,17,18]$. Elastase causes an irreversible change in the reactive centre loop of $\mathrm{CBG}$ changing the conformational state of the protein from a S-state ( $\mathrm{S}$ for stressed) into a $\mathrm{R}$-state ( $\mathrm{R}$ for relaxed), thus lowering the affinity by approximately a factor $10[19,20,21]$. By $X_{C^{*}}$ we denote the concentration of free $\mathrm{CBG}^{*}, X_{F C^{*}}$ that of cortisol bound to $\mathrm{CBG}^{*}\left(\right.$ Cort: $\left.\mathrm{CBG}^{*}\right), X_{E}$ that of free elastase, and $X_{C E}$ that of elastase bound to CBG (CBG:NE).

The enzymatic conversion of $\mathrm{CBG}$ into $\mathrm{CBG}^{*}$ is assumed to follow MichaelisMenten kinetics with $k_{C E}^{+}$and $k_{C E}^{-}$being the association and dissociation rates, respectively, of the reversible $\mathrm{CBG:NE}$ complex-binding and $k_{C^{*}}^{+}$the catalytic rate describing the irreversible conversion of CBG:NE into CBG*.

By $X_{T}$ and $X_{P}$ we denote the concentration of testosterone (Tes) and progesterone (Prog), respectively, $X_{T C}$ and $X_{P C}$ those of testosterone and progesterone bound to CBG (Tes:CBG and Prog:CBG), respectively, ignoring potential binding to $\mathrm{CBG}^{*}$. Similar, $X_{T A}$ and $X_{P A}$ denote concentrations of testosterone and progesterone bound to albumin (Tes:Alb and Prog:Alb), respectively.

By $k_{F s}$ we denote the secretion rate of cortisol into the bloodstream by the adrenal glands. The clearing rate of cortisol will be denoted $k_{F e}$. Secretion and elimination of CBG will be denoted $k_{C s}$ and $k_{C e}$, respectively, whereas $k_{C^{*} e}$ denote the clearing rate of $\mathrm{CBG}^{*}$. The fast production rate, $k_{E s}$, for elastase and elimination rate, $k_{E e}$, are also considered. Clearing rates for the other substances will be ignored since they are supposed to be much smaller $[22]$.

The association and dissociation rate constants of steroids and their transport proteins are denoted with a plus and a minus, respectively, e.g. the association and dissociation rate constants of cortisol and CBG are denoted $k_{F C}^{+}$and $k_{F C}^{-}$, respectively. We use the symbol $\emptyset$ for the pool of substances, commonly known as sinks or sources, assuming that the concentrations of substances in this pool do not affect the system. 
- Cort+CBG $\underset{k_{F C}^{-}}{\stackrel{k_{F C}^{+}}{\rightleftharpoons}}$ Cort:CBG

- Cort $+\mathrm{CBG}^{*} \underset{k_{F C^{*}}^{-}}{\stackrel{k_{F C^{*}}^{+}}{\rightleftharpoons}}$ Cort:CBG*

- Cort $+\mathrm{Alb} \underset{k_{F A}^{-}}{\stackrel{k_{F A}^{+}}{\rightleftharpoons}}$ Cort:Alb

- $\emptyset \stackrel{k_{F s}}{\longrightarrow}$ Cort $\stackrel{k_{F e}}{\longrightarrow} \emptyset$

- $\emptyset \stackrel{k_{C s}}{\longrightarrow} \mathrm{CBG} \stackrel{k_{C e}}{\longrightarrow} \emptyset$

- Tes+CBG $\underset{k_{T C}^{-}}{\stackrel{k_{T C}^{+}}{\rightleftharpoons}}$ Tes:CBG

- Tes+Alb $\underset{k_{T A}^{-}}{\stackrel{k_{T A}^{+}}{\rightleftharpoons}}$ Tes:Alb

- Prog+CBG $\underset{k_{P C}^{-}}{\stackrel{k_{P C}^{+}}{\rightleftharpoons}}$ Prog:CBG

- Prog + Alb $\underset{k_{P A}^{-}}{\stackrel{k_{P A}^{+}}{\rightleftharpoons}}$ Prog:Alb

- $\mathrm{CBG}+\mathrm{NE} \underset{k_{C E}^{-}}{\stackrel{k_{C E}^{+}}{\longrightarrow}} \mathrm{CBG}: \mathrm{NE} \stackrel{k_{C^{*}}^{+}}{\longrightarrow} \mathrm{CBG}^{*}+\mathrm{NE}$

- $\mathrm{CBG}^{*} \stackrel{k_{C^{*} e}}{\longrightarrow} \emptyset$

- $\emptyset \stackrel{k_{E s}}{\longrightarrow} \mathrm{NE} \stackrel{k_{E e}}{\longrightarrow} \emptyset$

Box 2.1.1 The reactions of the bio-chemical system controlling cortisols dynamic in the blood including the dynamics of cortisol (Cort), Corticosteroid-binding globulin on uncleaved and cleaved form ( $\mathrm{CBG}$ and $\mathrm{CBG}^{*}$, respectively), albumin (Alb), progesterone (Prog), testosterone (Tes), and neutrophil elastase (NE). The small ks denote the rate constants of the reactions. A common symbol $\emptyset$ denotes the sources of CBG, Cort, and NE and elimination products of CBG, $\mathrm{CBG}^{*}$, Cort, and $\mathrm{NE}$, since the concentrations of substances in this pool are assumed not to affect the system. The potential binding of Prog and Tes to $\mathrm{CBG}^{*}$ is ignored as well as the synthesis and clearing of Alb, Prog and Tes. 


\subsubsection{A static model by equilibrium considerations}

Assuming law of mass action and an equilibrium between the elimination and enzymatic synthesis of $\mathrm{CBG}^{*}$ gives,

$$
k_{C^{*} e} \cdot X_{C^{*}}=k_{C^{*}}^{+} \cdot X_{C E}
$$

Making a standard quasi steady state approximation, where the concentration of CBG:NE is assumed constant compared to the changes in the concentrations of other species, gives,

$$
X_{C E}=\frac{k_{C E}^{+}}{k_{C E}^{-}+k_{C^{*}}^{+}} \cdot X_{E} \cdot X_{C}
$$

Combining equation 1 and 2 results in,

$$
X_{C^{*}}=\frac{k_{C^{*}}^{+}}{k_{C^{*} e}} K_{M_{C E}}^{-1} \cdot X_{E} \cdot X_{C}
$$

where $K_{M_{C E}}$ is the Michaelis-Menten constant $K_{M_{C E}}=\frac{k_{C E}^{-}+k_{C^{*}}^{+}}{k_{C E}^{+}}$.

From equation 3 it follows that the fraction $X_{C^{*}} / X_{C}$ becomes proportional to $X_{E}$,

$$
\frac{X_{C^{*}}}{X_{C}}=\frac{X_{E}}{K_{C E}}
$$

with an approximated 'equilibrium dissociation constant' $K_{C E}=\frac{k_{C^{*}}}{k_{C^{*}}^{+}} K_{M_{C E}}$.

Using the law of mass action and assuming equilibrium we obtain ordinary equilibrium relations from the rest of the chemical reactions,

$$
\begin{aligned}
X_{F} \cdot X_{C} & =K_{F C} \cdot X_{F C} \\
X_{F} \cdot X_{C^{*}} & =K_{F C^{*}} \cdot X_{F C^{*}} \\
X_{F} \cdot X_{A} & =K_{F A} \cdot X_{F A} \\
X_{T} \cdot X_{C} & =K_{T C} \cdot X_{T C} \\
X_{T} \cdot X_{A} & =K_{T A} \cdot X_{T A} \\
X_{P} \cdot X_{C} & =K_{P C} \cdot X_{P C} \\
X_{P} \cdot X_{A} & =K_{P A} \cdot X_{P A}
\end{aligned}
$$

with $K_{F C}=\frac{k_{F C}^{-}}{k_{F C}^{+}}, K_{F C^{*}}=\frac{k_{F C^{*}}^{-}}{k_{F C^{*}}^{+}}, K_{F A}=\frac{k_{F A}^{-}}{k_{F A}^{+}}, K_{T C}=\frac{k_{T C}^{-}}{k_{T C}^{+}}, K_{T A}=\frac{k_{T A}^{-}}{k_{T A}^{+}}$, $K_{P C}=\frac{k_{P C}^{-}}{k_{P C}^{+}}, K_{P A}=\frac{k_{P A}^{-}}{k_{P A}^{+}}$being the equilibrium dissociation constants of the respective reactions. 
In addition to equations (4)-(5g), we have three source-sink balances,

$$
\begin{aligned}
& k_{F s}=k_{F e} \cdot X_{F} \\
& k_{C s}=k_{C e} \cdot X_{C} \\
& k_{E s}=k_{E e} \cdot X_{E}
\end{aligned}
$$

which are not used for finding the concentrations, but are needed in order to estimate the fractions between source and elimination constants at steady state.

Assuming conservation of total concentrations of the involved substances in addition to the above equilibrium assumptions, we obtain five coupled second order algebraic equations in five variables,

$$
\begin{aligned}
X_{C 0} & =X_{C}+X_{C^{*}}+X_{F C}+X_{F C^{*}}+X_{T C}+X_{P C}+X_{C E} \\
& =X_{C}\left(1+\frac{X_{E}}{K_{C E}}+\frac{X_{E}}{K_{M_{C E}}}+\frac{X_{F}}{K_{F C}}\left(1+\frac{X_{E} K_{F C}}{K_{C E} K_{F C^{*}}}\right)+\frac{X_{T}}{K_{T C}}+\frac{X_{P}}{K_{P C}}\right) \\
X_{F 0} & =X_{F}+X_{F C}+X_{F C^{*}}+X_{F A} \\
& =X_{F}\left(1+\frac{X_{C}}{K_{F C}}\left(1+\frac{X_{E} K_{F C}}{K_{C E} K_{F C^{*}}}\right)+\frac{X_{A}}{K_{F A}}\right) \\
X_{T 0} & =X_{T}+X_{T C}+X_{T A} \\
& =X_{T}\left(1+\frac{X_{C}}{K_{T C}}+\frac{X_{A}}{K_{T A}}\right) \\
X_{A 0} & =X_{A}+X_{F A}+X_{T A}+X_{P A} \\
& =X_{A}\left(1+\frac{X_{F}}{K_{F A}}+\frac{X_{T}}{K_{T A}}+\frac{X_{P}}{K_{P A}}\right) \\
X_{P 0} & =X_{P}+X_{P C}+X_{P A} \\
& =X_{P}\left(1+\frac{X_{C}}{K_{P C}}+\frac{X_{A}}{K_{P A}}\right)
\end{aligned}
$$

with $X_{C 0}$ being the total amount of CBG and $\mathrm{CBG}^{*}, X_{F 0}$ of cortisol, $X_{T 0}$ of testosterone, $X_{A 0}$ of albumin, and $X_{P 0}$ of progesterone in all forms.

By using relative variables $x_{C}=X_{C} / X_{C 0}, x_{F}=X_{F} / X_{F 0}, x_{T}=X_{T} / X_{T 0}$, $x_{A}=X_{A} / X_{A 0}$ and $x_{P}=X_{P} / X_{P 0}$ and grouping the parameters, the following 
dimensionless form in five variables and with 13 parameters is obtained,

$$
\begin{aligned}
p_{0} x_{C}+p_{C F} x_{C} x_{F}+p_{C T} x_{C} x_{T}+p_{C P} x_{C} x_{P} & =1 \\
x_{F}+p_{F C} x_{F} x_{C}+p_{F A} x_{F} x_{A} & =1 \\
x_{T}+p_{T C} x_{T} x_{C}+p_{T A} x_{T} x_{A} & =1 \\
x_{A}+p_{A F} x_{A} x_{F}+p_{A T} x_{A} x_{T}+p_{A P} x_{A} x_{P} & =1 \\
x_{P}+p_{P C} x_{P} x_{C}+p_{P A} x_{P} x_{A} & =1
\end{aligned}
$$

where $p_{0}=1+\frac{X_{E}}{K_{C E}}+\frac{X_{E}}{K_{M_{C E}}}=1+\frac{X_{E}}{K_{C E}}\left(1+\frac{k_{C^{*} e}}{k_{C^{*}}^{+}}\right) \approx 1+\frac{X_{E}}{K_{C E}} \approx 2.0, p_{F C}=$ $\frac{X_{C 0}}{K} \approx 19.9, p_{F A}=\frac{X_{A 0}}{K_{F A}} \approx 2.0, p_{T C}=\frac{X_{C 0}}{K_{T C}} \approx 1.4, p_{T A}=\frac{X_{A 0}}{K_{T A}} \approx 24.1$, $p_{A F}=\frac{X_{F 0}}{K_{F A}} \approx 6.1 \cdot 10^{-4}, p_{A T}=\frac{X_{T 0}}{K_{T A}} \approx 1.8 \cdot 10^{-4}, p_{C F}=\frac{X_{F 0}}{K} \approx 6.6 p_{C T}=$ $\frac{X_{T 0}}{K_{T C}} \approx 1.2 \cdot 10^{-2}, p_{C P}=\frac{X_{P 0}}{K_{P C}} \approx 0.12, p_{A P}=\frac{X_{P 0}}{K_{P A}} \approx 3.0 \cdot 10^{-4}, p_{P C}=$ $\frac{X_{C 0}}{K_{P C}} \approx 14.4$, and $p_{P A}=\frac{X_{A 0}}{K_{P A}} \approx 30.1$, with $K=\frac{K_{F C}}{1+\frac{X_{E} K_{F C}}{K_{C E} K_{F C^{*}}}} \approx 30.1$. The numerical values of the dimensionless parameters are calculated using default parameter values listed in section 2.2, which are based on the in the literature found values. Note that only 11 of the 13 parameters are independent, e.g. $p_{C F}=p_{F C} \frac{p_{T A} p_{A F} p_{C T}}{p_{A T} p_{F A} p_{T C}}$ and $p_{C P}=p_{P C} \frac{p_{C F} p_{F A} p_{A P}}{p_{P A} p_{A F} p_{F C}}$. Consequently, we only have 11 free parameters.

In equations $8 \mathrm{a}$ to $8 \mathrm{e}$, the following approximation is made: $p_{0}=1+\frac{X_{E}}{K_{C E}}+$ $\frac{X_{E}}{K_{M_{C E}}}=1+\frac{X_{E}}{K_{C E}}\left(1+\frac{k_{C^{*} e}}{k_{C^{*}}^{+}}\right) \approx 1+\frac{X_{E}}{K_{C E}}$, since the fraction $\frac{k_{C^{*}}}{k_{C^{*}}^{+}}$is assumed to be small $\left(<5 \cdot 10^{-5}\right.$, see section 2.2 .3$)$. When using the approximation the elastase concentration and the kinetic constants describing the enzyme's activity enters the model solely as the fraction of $X_{E}$ and $K_{C E}\left(n_{C E}=\frac{X_{E}}{K_{C E}}\right)$. We note that some of the other terms may be neglectable in size as well (see section 2.1.3 were a reduced version of the model is stated).

We may numerically solve equations (8a)-(8e) directly given the total concentrations $X_{C 0}, X_{A 0}, X_{F 0}, X_{T 0}$, and $X_{P 0}$ as input variables and $n_{C E}$ either as a parameter dependent on inflammation status, for known level of elastase as an input variable $n_{C E}=\frac{X_{E}}{K}$ or for known percentage of CBG on cleaved form $\left(n_{C C^{*}}\right)$ by an approximation (see section 2.2.3). Thereby we obtain a solution for the free substances $\left(X_{C}, X_{F}, X_{T}, X_{A}, X_{P}\right)$, and are afterwards able to calculate the concentrations of all complexes using equations (4)-(5g). We will term this model the static model. 


\subsubsection{A reduced version of the static model}

As the estimated values of the dimensionless parameters describe, the terms involving $p_{A F}, p_{A T}$ and $p_{A P}$ in equation (8d) are much smaller than $x_{A}$. This results in $x_{A} \approx 1$, which may be substituted into the equations $8 \mathrm{a}-8 \mathrm{c}$ and $8 \mathrm{e}$. Hence, solving this system of equations is equivalent to solving

$$
\begin{aligned}
& a_{0} x_{C}^{4}+a_{1} x_{C}^{3}+a_{2} x_{C}^{2}+a_{3} x_{C}+a_{4}=0 \\
& x_{F}=\frac{1}{1+p_{F A}+p_{F C} x_{C}} \\
& x_{T}=\frac{1}{1+p_{T A}+p_{T C} x_{C}} \\
& x_{P}=\frac{1}{1+p_{P A}+p_{P C} x_{C}} \\
& x_{A}=\frac{1}{1+p_{A F} x_{F}+p_{A T} x_{T}+p_{A P} x_{P}}
\end{aligned}
$$

in sequential order, where the constant coefficients $a_{0}, a_{1}, a_{2}, a_{3}$ and $a_{4}$ are expression in the earlier parameters and can be found in Appendix A. We will term this the reduced static model.

We notice that the present result demands solving a fourth order polynomial equation as is the case in the most recent paper by Nguyen et al. [3] despite the more details of our model such as including elastase, testosterone and progesterone. It should be noted when comparing the two equations that in Nguyen et al. [3] the roots of their fourth order polynomial equation is the free cortisol $\left(X_{F}\right)$, while the roots of the fourth order polynomial stated here is the relative variable describing the amount of uncleaved CBG in the free form $\left(x_{C}=X_{C} / X_{C 0}\right)$. The relative variable for free cortisol $\left(x_{F}=X_{F} / X_{F 0}\right)$ is calculated afterwards as stated in equation $9 \mathrm{~b}$.

Since the reduced static model is a fourth order polynomial, an analytical solution or "formula" can be found as was the case of the fourth, third and second order polynomial models of Nguyen et al. [3], Dorin et al. [2], and Coolens et al. [1], respectively. We have stated the only physiological relevant solution in appendix Appendix B. This formula can, as the earlier formulae $[3,2,1]$, be calculated in readily accessible programs such as EXCEL ${ }^{1}$.

\footnotetext{
${ }^{1}$ One should be aware of the accuracy of the calculations. Our implementation of
} 


\subsection{Parameter and input variable values}

\subsubsection{Dissociation constants}

As seen in table 1 literature found values of the dissociation constants varies considerably. Some of the variation might be due to difference in methodology. Most of the dissociation constants are determined in vitro. According to Dorin et al. [2] their fitting of $K_{F A}$ suggests that the affinity of albumin to cortisol might be higher in vivo than what is observed in vitro $[2]$.

While the affinity of CBG for cortisol and progesterone varies by temperature, the affinity of human albumin does not, but varies with $\mathrm{pH}$ [23]. The affinity of cleaved CBG is about 10-fold reduced compared to native CBG $[19,24]$.

the solution formulae by Nguyen et al. [3] and Dorin et al. [2] in MATLAB R2016b gave considerably less accurate solutions than solutions found with the inbuilt roots-function. Consequently, in the comparison of the different models' results we use the MATLAB inbuilt roots-function. 
Table 1: Equilibrium dissociation constants

\begin{tabular}{|c|c|c|}
\hline$K_{F C}=\frac{k_{F C}^{-}}{k_{F C}^{+}}$for Cort:CBG & $\begin{array}{l}0.71 \mathrm{nM} \\
11 \mathrm{nM} \\
13 \mathrm{nM} \\
18 \mathrm{nM} \\
19 \mathrm{nM} \\
21 \mathrm{nM} \\
25 \mathrm{nM} \\
32 \mathrm{nM} \\
\mathbf{3 3} \mathrm{nM} \\
39 \mathrm{nM} \\
240 \mathrm{nM} \\
292.2 \mathrm{nM} \\
\end{array}$ & $\begin{array}{l}{[24]^{\#}} \\
{[25]^{\#}} \\
{[7,26]^{\#}} \\
{[23]} \\
{[27]^{\#}} \\
{[28]^{\#}} \\
{[29,30,31]^{\#}} \\
{[19]} \\
{[32]} \\
{[33]^{\#}} \\
{[34]} \\
{[35]}\end{array}$ \\
\hline$K_{F C^{*}}=\frac{k_{F C^{*}}^{-}}{k_{F C^{*}}}$ for Cort:CBG* & $\begin{array}{l}6.25 \mathrm{nM} \\
292 \mathrm{nM} \\
1366.0 \mathrm{nM} \\
\mathbf{1 0}^{\mathbf{1 0}} \mathbf{K}_{F C} \\
\end{array}$ & $\begin{array}{l}{[24]^{\#}} \\
{[19]} \\
{[35]} \\
{[3,19,24]} \\
\end{array}$ \\
\hline$K_{F A}=\frac{k_{F A}^{-}}{k_{F A}^{+}}$for Cort:Alb & $\begin{array}{l}1.378 \cdot 10^{5} \mathrm{nM} \\
3.00 \cdot 10^{5} \mathrm{nM}(\text { at } \mathrm{pH} 7.8) \\
\mathbf{3 . 3 0} \cdot \mathbf{1 0}^{5} \mathrm{nM} \\
3.33 \cdot 10^{5} \mathrm{nM} \\
4.796 \cdot 10^{5} \mathrm{nM} \\
8.10 \cdot 10^{5} \mathrm{nM} \\
\left.9.00 \cdot 10^{5} \mathrm{nM} \text { (at } \mathrm{pH} 6.8\right)\end{array}$ & $\begin{array}{l}2] \\
{[23]} \\
{[32]} \\
{[7]^{\#}} \\
{[33]^{\#}} \\
{[36]} \\
{[23]}\end{array}$ \\
\hline$K_{T A}=\frac{k_{T A}^{-}}{k_{T A}^{+}}$for Tes:Alb & $\begin{array}{l}2.46 \cdot 10^{4} \mathrm{nM} \\
2.50 \cdot 10^{4} \mathrm{nM} \\
\mathbf{2 . 7 8} \cdot 10^{4} \mathrm{nM} \\
2.80 \cdot 10^{4} \mathrm{nM} \\
2.9 \cdot 10^{4}-3.1 \cdot 10^{4} \mathrm{nM} \\
4.00 \cdot 10^{4} \mathrm{nM} \\
\end{array}$ & $\begin{array}{l}{[37]^{\#}} \\
{[7]^{\#}} \\
{[38,39,40]^{\#}} \\
{[27]^{\#}} \\
{[41]^{\#}} \\
{[42]^{\#}}\end{array}$ \\
\hline$K_{T C}=\frac{k_{T C}^{-}}{k_{T C}^{+}}$for Tes:CBG & $\begin{array}{l}189 n M \\
417 \mathbf{n M} \\
667 n M\end{array}$ & $\begin{array}{l}{[7]^{\#}} \\
{[30]^{\#}} \\
{[40]^{\#}} \\
\end{array}$ \\
\hline$K_{P C}=\frac{k_{P C}^{-}}{k_{P C}^{+}}$for Prog:CBG & $\begin{array}{l}11.1 \mathrm{nM} \\
38 \mathrm{nM} \\
\mathbf{4 1 . 7} \mathbf{n M} \\
85 \mathrm{nM}\end{array}$ & $\begin{array}{l}{[43]^{\#}} \\
{[31]^{\#}} \\
{[7,26]^{\#}} \\
{[23]}\end{array}$ \\
\hline$K_{P A}=\frac{k_{P A}^{-}}{k_{P A}^{+}}$for Prog:Alb & $\begin{array}{l}\mathbf{1 . 6 7 \cdot 1 0 ^ { 4 }} \mathbf{n M} \\
9.0 \cdot 10^{4} \mathrm{nM}(\text { at } \mathrm{pH} 7.8) \\
\left.20.0 \cdot 10^{4} \mathrm{nM} \mathrm{nM} \mathrm{(at} \mathrm{pH} 6.8\right)\end{array}$ & $\begin{array}{l}{[7]^{\#}} \\
{[23]} \\
{[23]}\end{array}$ \\
\hline
\end{tabular}

Equilibrium dissociation constants with references. Some dissociation constants are calculated from the corresponding association constant marked by a ${ }^{\#}$ at the references. Our choice of defeault parameter values are emplhasized in bold. 


\subsubsection{Steroid and transport protein concentrations}

The reported values for CBG varies considerably (See table S.1). The normal range is wide as reported by Lewis et al. [44] 312 to $1324 \mathrm{nM}$ for 20 normal individuals [44]. Cameron et al. [23] defines three examples of CBG concentration, i.e. high level at $1300 \mathrm{nM}$, normal at $600 \mathrm{nM}$, and low at 300 $\mathrm{nM}$ [23].

It is possible to measure specifically the uncleaved CBG $\left(C_{t o t}\right)$ with antibodies and afterwards get a relation of how much CBG is elastase cleaved by comparing these measurements with measurements made with other antibodies that binds to both the uncleaved and the elastase cleaved CBG [20] $\left(C_{t o t}^{*}=X_{C 0}-C_{t o t}\right)$. According to Lewis and Elder [45] significantly higher levels of CBG measured in both total and intact CBG are seen in women compared to men. The concentration of total and native CBG were $644 \pm 120$ $\mathrm{nM}$ and $438 \pm 113 \mathrm{nM}$, respectively, in women and $574 \pm 134 \mathrm{nM}$ and $379 \pm 131$ nM, respectively, for men [45]. For both sexes the ratio of cleaved CBG compared to total $\mathrm{CBG}, n_{C C^{*}}=\frac{C_{\text {tot }}^{*}}{X_{C 0}}$, is $0.30-0.35$ [45]. For sixteen normal individuals with corresponding levels of total and intact CBG reported in Lewis and Elder [20, figure 6a, p 292] the range of $n_{C C^{*}}$ is approximately 0 to 0.635 .

The concentration of albumin in the blood is much larger than CBG and the steroids included in the model. The normal range of albumin is $5.49 \cdot 10^{5}$ $\mathrm{nM}$ to $7.20 \cdot 10^{5} \mathrm{nM}$ [46]. However, the level is frequently decreased in elderly people [47].

The normal ranges of testosterone in plasma are 5.83 to $26.30 \mathrm{nM}$ in men and $<0.35$ to $3.12 \mathrm{nM}$ in women [48]. Lower levels of testosterone are seen in some older compared to younger men $(12.1 \pm 0.7 \mathrm{nM}$ vs $17.7 \pm 1.0 \mathrm{nM}$ in Plymate et al. [49]), though the same picture is not visible across all populations [50]. Testosterone concentration normal ranges vary considerably between different laboratories as reported in the resent review by Le et al. [51]. Le et al. [51] attributes some of the variation to the underlying population studies including participants with unknown medical histories [51]. However, also in studies such as Salonia et al. [11], where the participating female subjects were chosen so to be without sexual disorders, the range was wide [11]. Likewise, normal ranges for cortisol varies greatly. Pretorius et al. [4] attributes this not only to differences in the cohorts used to determine the ranges, but also to differences in the techniques used [4]. The assays for determining cortisol concentrations vary greatly [6]. Our findings in the liter- 
ature reported in table S.3, S.5, S.6 of the Supplementary materials suggest that the same might hold for progesterone.

The dynamic behaviour of especially cortisol [4], but also testosterone $[52,53,49]$ and progesterone $[11,54]$ should be taken into account. Cortisol levels have great intra-personal variability with a clear circadian rhythm as well as a faster ultradian rhythm with pulses every 1 to 2 hours [55]. The circadian rhythm of cortisol is present in some reference ranges, e.g. Aardal and Holm [56] reports 200 to $800 \mathrm{nM}$ as their serum cortisol reference range at 8 a.m., but $<300 \mathrm{nM}$ at 10 p.m. [56]. At 12 p.m. the concentration is even lower $<50 \mathrm{nM}[57]$.

It seems that a circadian rhythm in testosterone levels is present in some men $[52,53,49]$. In pregnant women a circadian rhythm has been observed with an inverse relationship to the cortisol rhythm $[54,58]$. In women not only progesterone, but also testosterone fluctuates during the menstrual cycle $[11]$.

In men the normal range of progesterone is 0.6 to $4.5 \mathrm{nM}$, while it for women varies from 0.6 to $4.8 \mathrm{nM}$ in the follicular phase and 5.4 to $85.9 \mathrm{nM}$ in the luteal phase of the menstrual cycle [8]. In pregnant women at term the concentration is approximately $541 \mathrm{nM}[12]$. CBG [59, 60], cortisol[13, 60], and testosterone concentrations [13] rise during pregnancy, while the concentration of albumin declines [61, 62].

Though the total level of CBG increases in pregnancy, the level of cleaved CBG does not, which results in a smaller ratio of cleaved CBG than normal $\left(n_{C C^{*}}=0.167\right)[60]$. The increase in CBG during pregnancy is attributed to a direct estrogen-induced rise in production. In women using estrogen-based combined oral contraceptive pills (COCP) the total CBG level is similarly increased $[63,60]$, but the level of cleaved $\mathrm{CBG}$ is also increased, though to a lesser degree $\left(n_{C C^{*}}=0.269\right.$ in $\mathrm{COCP}$ women vs $n_{C C^{*}}=0.433$ in the normal control women) [60].

\subsubsection{Elastase activity}

The activity of elastase is described by the input variable or parameter $n_{C E}=\frac{X_{E}}{K_{C E}}$ with $K_{C E}=\frac{k_{C^{*}}^{+}}{k_{C^{*}}} K_{M_{C E}}^{-1}$ as described in section 2.1.2 and 2.1.3.

The level of elastase has been found to be elevated in systemic inflammatory response syndrome (SIRS) patients [64] and in chronic obstructive pulmonary disease (COPD) patients [65]. Higher levels of neutrophil elastase activity have been found in mild and severe preeclampsia [66]. The complex 
of neutrophil elastase and the native elastase-inhibitor $\alpha_{1}$-antitrypsin (NE$\alpha_{1} \mathrm{AT}$ ) have in other studies been used as a measure of the release of free elastase and the activity of neutrophils in condition of inflammation [67]. Elevated levels of NE- $\alpha_{1}$-AT have been found in e.g. patients with Crohns disease (CD), ulcerative colitis (UC) [68], food hypersensitivity [69], and intermediate uveitis (IU) [67].

The magnitude of the increase in elastase activity differs between diseases and reported studies from a less than 2 fold increase in [66], 2-3 fold increase in Polańska et al. [67] and Pawlica-Gosiewska et al. [68], 4 fold increase in Zbikowska-Gotz et al. [69] and up to more than 10 fold increase in Kodama et al. [64]. In 20 normal subjects Donnelly et al. [70] found a mean of 0.631 $\mathrm{nM}$ and a range of 0.31 to $1.73 \mathrm{nM}$ elastase [70]. In multiple trauma patients the concentration ranged from 0.725 to $23.051 \mathrm{nM}[70]$.

The elimination constant $k_{C^{*}}$ for human $\mathrm{CBG}^{*}$ is reported to be the same as for uncleaved CBG in a study done in rabbits [17]. In humans the half life of CBG is approximately 5 days (range measured 4.6 to 6.0 days in five subjects) [71], i.e. $k_{C^{*} e}=k_{C e} \approx 1 \cdot 10^{-4} \mathrm{~min}^{-1}$.

It has been difficult to find measurements of $K_{M_{C E}}$ and $k_{C^{*}}^{+}$in the literature. For other substrates in the literature reported values of the MichaelisMenten constant of human neutrophil elastase range from $1 \cdot 10^{3}$ to 3.7 . $10^{6} \mathrm{nM}$ and the catalytic constant $\left(k_{c a t}\right)$ from 2.4 to $288000 \mathrm{~min}^{-1}[72]$. As an example the $K_{M}=1.4 \cdot 10^{5} \mathrm{nM}$ and the catalytic constant $k_{\text {cat }}=$ $1.0 \cdot 10^{3} \mathrm{~min}^{-1}$ for elastase's cleavage of methoxysuccinyl-Ala-Ala-Pro-Val4-nitroanilide [73]. However, CBG as opposed to methoxysuccinyl-Ala-AlaPro-Val-4-nitroanilide and a lot of other elastase substrates after cleavage does not inhibit the further activity of elastase and Hammond et al. [74] describe the cleavage of CBG by elastase as extremely efficient [74] and SumerBayraktar et al. [75] the reaction as fast.

For unknown elastase level, but known $C_{t o t}$ and $C_{\text {tot }}^{*}$, we will make an approximated input variable expression. From equation 4 , we have that $\frac{X_{E}}{K_{C E}}$ is equivalent to the a priori unknown fraction of $X_{C^{*}}$ to $X_{C}$. However, leaving out the progesterone, testosterone and elastase bound fractions we approximately have $C_{t o t} \approx X_{C}\left(1+\frac{X_{F}}{K_{F C}}\right)$ and $C_{t o t}^{*} \approx X_{C} \frac{X_{E}}{K_{C E}}\left(1+\frac{X_{F}}{K_{F C^{*}}}\right)$. Looking at the fraction of $C_{t o t}^{*}$ to $C_{t o t}$ and isolating $\frac{X_{E}}{K_{C E}}$ we get:

$$
\frac{X_{E}}{K_{C E}}=\frac{\left(1+\frac{X_{F}}{K_{F C}}\right)}{\left(1+\frac{X_{F}}{K_{F C^{*}}}\right)} \frac{C_{t o t}^{*}}{C_{t o t}}=k_{n c e} \frac{C_{t o t}^{*}}{C_{t o t}}=k_{n c e} \frac{n_{C C^{*}}}{1-n_{C C^{*}}}
$$


The factor $k_{n c e}=\frac{\left(1+\frac{X_{F}}{K_{F C}}\right)}{\left(1+\frac{X_{F}}{K_{F C}}\right)}$ is not a true constant, since it depends on the level of free cortisol, $X_{F}$. The range of $X_{F}$ in normal subjects is 5.5 to $38.9 \mathrm{nM}$ in the morning [44], while higher levels can be found in e.g. sepsis [76] (for more values found in the literature see table S.3 in Supplementary Materials). Default values for the dissociation constants are $K_{F C}=33 \mathrm{nM}$ and $K_{F C^{*}}=10 \cdot K_{F C}$ (see table 1$)$. This being so, we may use $k_{n c e}=2$ as a gross estimate.

\subsubsection{Default parameter and input-variable values}

In section 3 the sensitivities and performances of the static model (see section 2.1.2) and the reduced static model (see section 2.1.3) are compared to the performances of the models presented in Coolens et al. [1], Dorin et al. [2], Nguyen et al. [3].

The input variable $X_{C 0}$ and $X_{F 0}$ as well as the parameter $K_{F C}$ (in Coolens et al. [1] as the association constant, $\frac{1}{K_{F C}}$ ) are present in all five models. $X_{A 0}$ and $K_{F A}$ are included in all models except Coolens et al. [1].

Nguyen et al. [3] and our models include $K_{F C^{*}}$. A parameter $n_{C C^{*}}=\frac{C^{*} t o t}{X_{C 0}}$ describes the percentage of $\mathrm{CBG}$ on cleaved form. The total concentrations of cleaved and uncleaved CBG $\left(C^{*}{ }_{t o t}\right.$ and $C_{t o t}$, respectively) are only explicitly present in the model by Nguyen et al. [3], but with the approximation $n_{C E} \approx$ $\frac{k_{n e} C_{t o t}^{*}}{C_{t o t}}=\frac{k_{n e} \cdot n_{C C^{*}}}{1-n_{C C^{*}}}$ enters our model as well. However, with our model one can chose to explicitly include the activity of elastase measured as $n_{C E}=\frac{X_{E}}{K_{C E}}$. Additionally, our models include $X_{T 0}$ and $X_{P 0}$ as well as $K_{T C}, K_{P C}, K_{T A}$, and $K_{P A}$.

The chosen default values of the equilibrium dissociation constants are emphasised in bold in table 1 and the input variables in table 2. For some analysis, including the sensitivity analysis, the total concentrations of testosterone and progesterone are set to the maximums of their normal ranges in order to investigate the maximal effect, i.e. $X_{T 0}=26.30 \mathrm{nM}$ (men [48]), and $X_{P 0}=85.9 n M$ (women in luteal phase [8]). We use the estimate $n_{C E}=\frac{X_{E}}{K_{C E}} \approx k_{n c e} \frac{n_{C C^{*}}}{1-n_{C C^{*}}}$ when a priori values for the concentrations of $\mathrm{CBG}$ and $\mathrm{CBG}^{*}$ are known from measurements and the default $k_{n c e}=2$. When not known, the default ratio of cleaved CBG to total CBG is set to $n_{C C^{*}}=0.325$.

These default values are in reasonable agreement with the dissociation constants listed in table 1 in section 2.2.1, the normal ranges described in section 2.2.2, and the additional values and normal ranges listed in table S.1 
Table 2: Default input variable values as well as representative population groups

\begin{tabular}{|l|l|l|l|l|l|l|l|}
\hline & Default & YM & OM & YWL & YWF & PW3T & COCP \\
\hline$X_{C 0}[\mathrm{nM}]$ & $\mathbf{6 0 0}$ & 574 & 574 & 644 & 644 & 877 & 1093 \\
\hline$X_{A 0}\left[\cdot 10^{4} \mathrm{nM}\right]$ & $\mathbf{6 7}$ & 70.1 & 66.2 & 70.1 & 70.1 & 58.4 & 70.1 \\
\hline$X_{F 0}[\mathrm{nM}]$ & $\mathbf{2 0 0}$ & 210 & 210 & 210 & 210 & 630 & 630 \\
\hline$X_{P 0}[\mathrm{nM}]$ & $\mathbf{5 ( 8 5 . 9 )}$ & 1.5 & 1.5 & 85.9 & 1.5 & 575 & 0.86 \\
\hline$X_{T 0}[\mathrm{nM}]$ & $\mathbf{5 ( 2 6 . 3 )}$ & 26.3 & 12.1 & 1.4 & 1.4 & 2.8 & 1.4 \\
\hline$n_{C C^{*}}[]$ & $\mathbf{0 . 3 2 5}$ & 0.343 & 0.343 & 0.343 & 0.343 & 0.167 & 0.269 \\
\hline$n_{C E} \approx \frac{2 \cdot n_{C C^{*}}}{1-n_{C C^{*}}}[]$ & $\mathbf{0 . 9 6 3}$ & 1.044 & 1.044 & 1.044 & 1.044 & 0.401 & 0.736 \\
\hline
\end{tabular}

Default input variable values are shown in bold. For some analysis of $X_{P 0}$ and $X_{T 0}$ the values stated in the brackets corresponding to the maximum in the normal range for young women and men are used. Additionally, estimated $24 \mathrm{~h}$ mean input variable values in different population groups are shown; $\mathrm{YM}=$ young men, $\mathrm{OM}=$ old men, $\mathrm{YWL}=$ young women in the luteal phase, $\mathrm{YWF}=$ young women in the folicular phase, $\mathrm{PW}$ T $=$ pregnant women in the 3rd trimester, and $\mathrm{COCP}=$ women tacking estrogen-based combined oral contraceptive pills. The values are chosen by the relations described in section 2.2 .2 and from values found in $[77,63,45,60,61,78,58,13,10,12,11,79,49,14,80,48]$.

to S.6 in Supplementary materials.

In section 3.2 we investigate the effect of individually varying the parameters and the input variables in the normal range reported in the literature. In 3.4 the combined effect of varying the input variables as typically seen in individuals of different age and gender is shown with estimated input variable values for a typical young man (YM), old man (OM), young woman in the luteal phase (YWL), young woman in the folicular phase (YWF), pregnant woman in the third trimester (PW3T), and a woman using estrogen-based combined oral contraceptive pills (COCP) (see table 2).

\subsection{Data of four specific subjects}

In Lewis and Elder [20] data of total CBG, native CBG and free cortisol levels measured by ultrafiltration/ligand binding can be found for four patients (see Lewis and Elder [20, table 1, p.293]). Two of these patients were termed discordant (sample Discordant 1 and 2), since the levels of intact CBG were less than $50 \%$ of total plasma CBG levels. For the rest of the samples that Lewis and Elder [20] looked at, the differences between total and intact CBG levels were less than 20\%. Hence, these samples were termed concordant and the data of two of these (sample Concordant 1 and 2) were shown in the same table as the discordant data for comparison [20]. 
Nguyen et al. [3] used the data of the four patients found in Lewis and Elder [20] to compare the predictability of Coolens [1], the cubic [2] and their own formulae. Since the albumin concentrations for these samples are not measured, Nguyen et al. [3] made two cases for albumin affinity with $K_{F A}=330,000 \mathrm{nM}$ (case A) and $K_{F A}=137,800 \mathrm{nM}$ (case B). Using the ratio of total albumin to its dissociation constant $N=X_{A 0} / K_{F A}=1.74$ of Coolens et al. [1], the total albumin concentrations of case A and B are $574,200 \mathrm{nM}$ and 239,772 nM, respectively. Our models predictions for these two cases are tested with $n_{C E}$ approximated by $n_{C E} \approx \frac{k_{n c e} C_{t o t}^{*}}{C_{t o t}}$ with the default $k_{n c e}=2$.

In a third case $\mathrm{C}$ with albumin affinity and concentration as in case $\mathrm{A}$, the activity of neutrophil elastase $n_{C E}$ is varied individually for each of the four subjects. In a fourth case $\mathrm{D}$, the parameter $k_{n c e}$ is fitted as one value common for the four subjects, but different from the default value $k_{n c e}=2$.

\subsection{Method of sensitivity analysis}

The change in concentration of free cortisol as a result of a $\pm 1 \%$ variation from the default value of a given parameter $\Theta_{i}$ and normalised to the change in the parameter value is used as a measure of absolute sensitivity $\left(s_{a}\left(\Theta_{i 1 \%}\right)=\frac{\Delta X_{F}\left(\Theta_{i \pm 1 \%}\right)}{\Delta \Theta_{i \pm 1 \%}}=\frac{X_{F}\left(1.01 \Theta_{i_{\text {default }}}\right)-X_{F}\left(0.99 \Theta_{i_{\text {def }} \text { aul }}\right)}{\left.0.02 \Theta_{i_{\text {def fault }}}\right)}\right)$. The sensitivity analysis is local, since it depends on the parameter values chosen as default values.

We will investigate the sensitivity of our models for both $n_{C C^{*}}$ and $n_{C E}$ by using the $n_{C C^{*}}$-dependent approximation of $n_{C E}$ for all runs except for the one where the sensitivity towards $n_{C E}$ is investigated.

The relative sensitivities is considered as well $\left(s_{r}\left(\Theta_{i 1 \%}\right)=\frac{\Theta_{i_{\text {default }}}}{X_{F}\left(\Theta_{\left.i_{\text {default }}\right)}\right)}\right.$. $\left.\frac{\Delta X_{F}\left(\Theta_{i \pm 1 \%}\right)}{\Delta \Theta_{i \pm 1 \%}}\right)$, since the sizes of the parameters vary greatly compared to each other (see section 2.2.4).

Furthermore, the differences in variation of the individual parameters are considerable (see section 2.2.1 to 2.2.4). Hence, we define a measure, where the sensitivities are normalised by a factor $\left(\Delta_{\text {literature }}\right)$ determined by the variation of the parameters seen in the literature $\left(s_{l}\left(\Theta_{i 1 \%}\right)=\Delta_{\text {literature }} \frac{\Delta X_{F}\left(\Theta_{i \pm 1 \%}\right)}{\Delta \Theta_{i \pm 1 \%}}\right)$. For the dissociation constants the differences between the largest and smallest values reported are used as $\Delta_{\text {literature }}$, while the differences between the upper and lower limits of the normal ranges with both genders considered are used for the input variables, which gives $\Delta_{\text {literature }}\left(X_{C 0}\right)=1012 \mathrm{nM}$, $\Delta_{\text {literature }}\left(X_{F 0}\right)=750 \mathrm{nM}, \Delta_{\text {literature }}\left(X_{A 0}\right)=1.71 e 5 \mathrm{nM}, \Delta_{\text {literature }}\left(n_{C C^{*}}\right)=$ 
$0.635, \Delta_{\text {literature }}\left(n_{C E}\right), \Delta_{\text {literature }}\left(X_{T 0}\right)=26.3 \mathrm{nM}, \Delta_{\text {literature }}\left(X_{P 0}\right)=85.9$ $\mathrm{nM}, \Delta_{\text {literature }}\left(K_{F C}\right)=\Delta_{\text {literature }}\left(K_{F C^{*}}\right)=291 \mathrm{nM}, \Delta_{\text {literature }}\left(K_{F A}\right)=7.62 e 5$ $\mathrm{nM}, \Delta_{\text {literature }}\left(K_{T C}\right)=478 \mathrm{nM}, \Delta_{\text {literature }}\left(K_{T A}=1.54 e 4 \mathrm{nM}, \Delta_{\text {literature }}\left(K_{P C}\right)=\right.$ $73.9 \mathrm{nM}$, and $\Delta_{\text {literature }}\left(K_{P A}\right)=18.33 e 4 \mathrm{nM}$.

\section{Results}

\subsection{Sensitivity analysis}

All five models show an across models similar sensitivity towards the parameters included in multiple models, e.g. $s_{a}\left(K_{F C}\right)$ is $0.342,0.356,0.283$, and 0.296 for our static model, Nguyen et al. [3], Dorin et al. [2], and Coolens et al. [1], respectively (see figure 1). The reduced static model shows close to identical sensitivities to the static model. Ranking the absolute values of the absolute sensitivities $\left(s_{a}\right)$ for the parameters of our static model, the ascending order of the parameters is: $K_{T A}, X_{A 0}, K_{F A}, K_{P A}, K_{T C}, X_{T 0}$, $X_{P 0}, K_{F C^{*}}, K_{P C}, X_{C 0}, X_{F 0}, K_{F C}, n_{C E}, n_{C C^{*}}$. The same sequence applies to sensitivities of the other models towards common parameters.

If we divide the ranked absolute sensitivities into four categories; our static model shows low sensitivity towards $K_{T A}, X_{A 0}, K_{F A}, K_{P A}, K_{T C}$, and $X_{T 0}\left(5.13 \cdot 10^{-7}\right.$ to $\left.5.84 \cdot 10^{-4}\right)$, is sensitive towards $X_{P 0}, K_{F C^{*}}$, and $K_{P C}$ $\left(3.19 \cdot 10^{-3}\right.$ to $\left.5.78 \cdot 10^{-3}\right)$, very sensitive towards $X_{C 0}, X_{F 0}, K_{F C}\left(2.71 \cdot 10^{-2}\right.$ to $3.42 \cdot 10^{-1}$ ), and extremely sensitive towards $n_{C E}$ and $n_{C C^{*}}$ (5.05 to 22.15).

As seen by the sensitivity for the parameter $n_{C E}$, our model is sensitive towards including elastase. The seemingly greater sensitivity towards changing $n_{C C^{*}}$ is due to the approximation $n_{C E}=\frac{X_{E}}{K_{C E}} \approx \frac{2 \cdot n_{C C^{*}}}{1-n_{C C^{*}}}$ (see section 2.4). Hence, a percentage change of $n_{C C^{*}}$ gives a larger percentage change than changing $n_{C E}$ directly by the same percentage. The approximation will be investigated further in section 3.2.4 and 3.3.

All five models are very sensitive towards the three input variables and parameters included in the original model by Coolens et al. [1], i.e. native CBG-cortisol dissociation constant $\left(K_{F C}\right)$, the total concentration of CBG $\left(X_{C 0}\right)$, and the total concentrations of cortisol $\left(X_{F 0}\right)$.

Looking at the parameters describing the influence of progesterone and testosterone $\left(K_{T A}, K_{P A}, K_{T C}, X_{T 0}, X_{P 0}, K_{P C}\right)$, which are unique for our static model compared to the models by Coolens et al. [1], Dorin et al. [2], Nguyen et al. [3], our static model have similar sensitivities towards $X_{P 0}$ and $K_{P C}$ as towards the dissociation constant of cortisol and cleaved CBG 
$\left(K_{F C^{*}}\right)$. The sensitivities towards the testosterone parameters $\left(K_{T A}, K_{T C}\right.$, $\left.X_{T 0}\right)$ are all low $\left(<10^{-3}\right)$.

The models by Dorin et al. [2], Nguyen et al. [3] and ours all include an input variable $X_{A 0}$ describing the concentration of albumin and the dissociation constant $K_{F A}$. In our model parameters describing the binding of progesterone and testosterone are included as well. Interestingly, all of the parameters describing albumin's interaction with the steroids fall in the low absolute sensitivity category. If we take into account the size difference of the parameters by looking at relative sensitivities $\left(s_{r}\left(\Theta_{i 1 \%}\right)\right)$, the relative sensitivities for albumin's influence are similar to the relative sensitivities towards the parameters describing CBG on different forms (for the static model the ascending order is $K_{T A}, K_{T C}, X_{T 0}, K_{P A}, K_{P C}, X_{P 0}, K_{F C^{*}}, K_{F A}, X_{A 0}, n_{C E}$, $n_{C C^{*}}, K_{F C}, X_{C 0}, X_{F 0}$, see figure S.1a in Supplementary materials). However, when we take into account the inter- and intra-individual variations in the parameters and input variables by looking at the sensitivities related to the variance of the parameters seen in the literature $\left(s_{l}\left(\Theta_{i 1 \%}\right)\right)$, the importance of varying the size of $X_{A 0}$ in the normal range seems to be small (for the static model the ascending order is $K_{T A}, X_{T 0}, K_{T C}, X_{P 0}, K_{P C}, K_{F C^{*}}, X_{A 0}$, $K_{P A}, K_{F A}, n_{C C^{*}}, n_{C E}, X_{C 0}, X_{F 0}, K_{F C}$, see figure S.1b in Supplementary materials). 


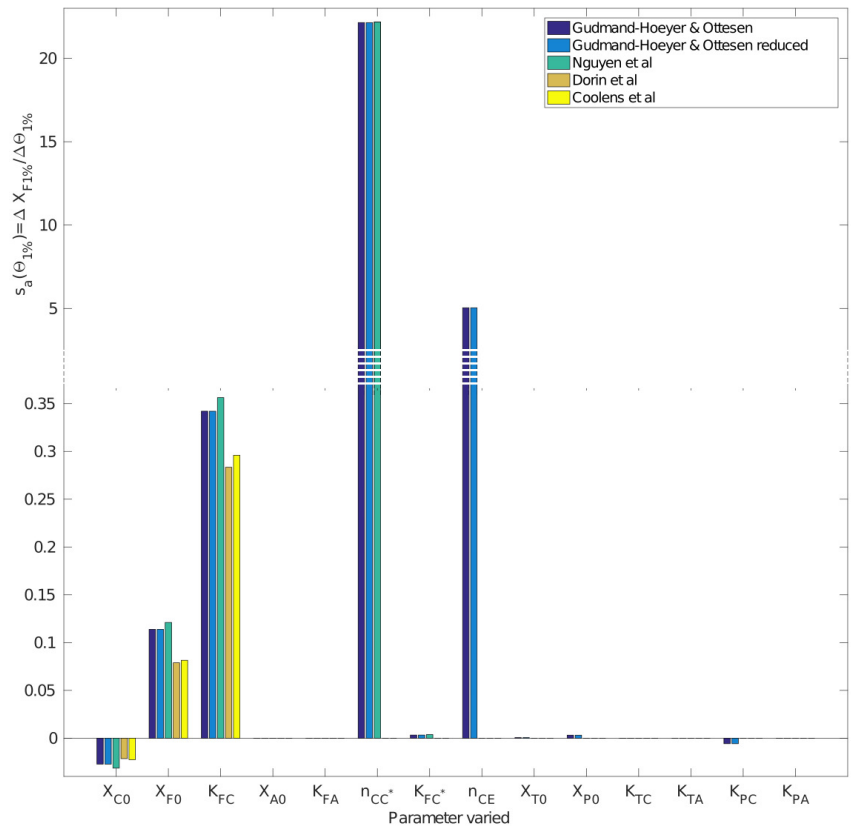

Figure 1: The absolute sensitivities towards common parameters are similar across the five models. Absolute sensitivities of the static model (Gudmand-Hoeyer \& Ottesen) and reduced static model (Gudmand-Hoeyer \& Ottesen reduced) compared to the sensitivities of the models by Nguyen et al. [3], Dorin et al. [2], and Coolens et al. [1] to a $1 \%$ variation in the parameter values $\left(s_{a}\left(\Theta_{i 1 \%}\right)\right)$. Change in scale of $s_{a}\left(\Theta_{i 1 \%}\right)$ is indicated as dotted axis and bars. 


\subsection{Individual variation of parameters and input variable values}

\subsubsection{Variation in total cortisol concentrations}

The influence of varying total cortisol $\left(X_{F 0}\right)$ in the range of 0 to 2000 $\mathrm{nM}$ on calculated measures of free cortisol is shown in figure 2. In figure 2a the calculated cortisol on different forms for the static model is shown, while calculated free cortisol for the static model and the reduced static model as well as the models in Nguyen et al. [3], Dorin et al. [2], and Coolens et al. [1] are shown in figure $2 \mathrm{~b}$ for comparisons. Figure $2 \mathrm{c}$ and $2 \mathrm{~d}$ show the results in absolute concentrations $[\mathrm{nM}]$. The results of the static model and the reduced static model are close to identical (see figure $2 \mathrm{~b}$ and $2 \mathrm{~d}$ ). All parameters and input variables beside $X_{F 0}$ are kept as their default values (see section 2.2.4).

All models in figure $2 \mathrm{~b}$ give a similar sigmoid relation in calculated free cortisol percentage $\left(x_{F}\right)$ for the variation of $X_{F 0}$, though with different maximum value, slope and turning point. The model by Coolens et al. [1] starts at the lowest free cortisol percentage, but end up with the highest value. The results of the static and the reduced static model is practically identical.

The circadian rhythm of the cortisol level is indicated on the figure by depicting maximum levels for 8 a.m., 10 p.m. and 12 p.m. normal ranges (800 nM, $300 \mathrm{nM}$ [56] and $50 \mathrm{nM}$ [57], respectively). Though some circadian variation can be present in some of the other input variables (CBG, progesterone and testosterone), cortisol is by far the substance with greatest intra-personal variation. Hence, figure 2 shows that there is a great variation of the level of free cortisol both measured in percentage and in absolute concentration during a normal day. Figure $2 \mathrm{c}$ and $2 \mathrm{~d}$ show that the actual changes measured in $\mathrm{nM}$ are large and that the CBG-bound fractions $\left(X_{F C}\right.$

and $X_{F C^{*}}$ ) both get close to saturation levels in the cortisol peak hours of the day ( 8 a.m. max level). Meanwhile the fraction of cortisol bound to albumin $\left(X_{F A}\right)$ still increases at $X_{F 0}=2000 \mathrm{nM}$. 


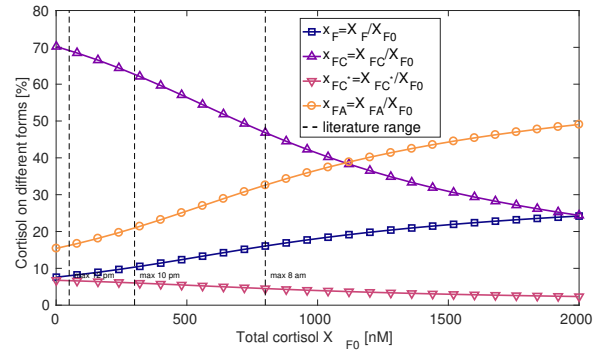

(a)

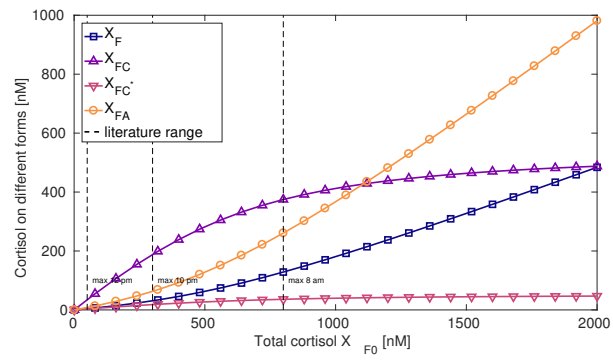

(c)

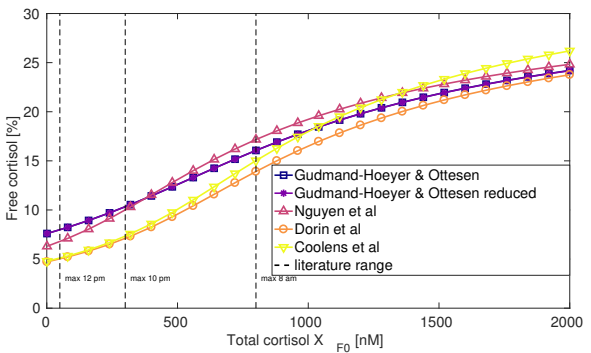

(b)

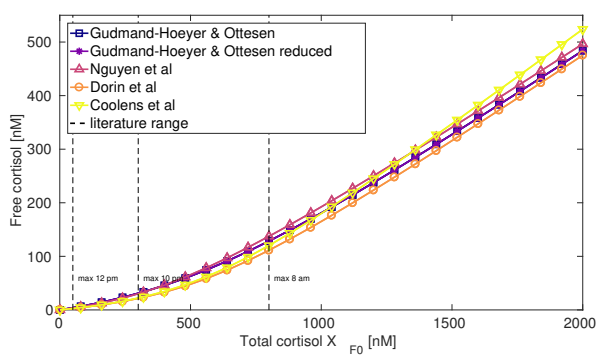

(d)

Figure 2: The static, the reduced static and the models of [1], [2], and [3] show qualitatively similar responses to change in total cortisol, but difference in actual concentrations. The total concentration of cortisol $\left(X_{F 0}\right)$ is varied. Maximum $X_{F 0}$ levels for 12 p.m., 10 p.m., and 8 a.m. normal ranges are indicated by vertical dotted lines (50 nM [57], 300 $\mathrm{nM}, 800 \mathrm{nM}$ [56], respectively). a) The calculated concentrations of cortisol in all forms as percentage of total cortisol given is shown for the static model (Gudmand-Hoeyer \& Ottesen). b) The calculated concentrations of cortisol in the free form for the static model (Gudmand-Hoeyer \& Ottesen) and the reduced static model (Gudmand-Hoeyer \& Ottesen reduced) as well as the models in Nguyen et al. [3] (Nguyen et al), Coolens et al. [1] (Coolens et al), and Dorin et al. [2] (Dorin et al) are shown as percentages of total cortisol. c) As 2a, but in absolute concentrations [nM]. d) As 2b, but in absolute concentrations $[\mathrm{nM}]$. The reduced static and the static model show close to identical results. 


\subsubsection{The impact of albumin variation}

In figure 3 the total albumin concentration $\left(X_{A 0}\right)$ and the albumin-cortisol dissociation constant $\left(K_{F A}\right)$, respectively, are varied.

As seen on figure $2 \mathrm{a}$ and even clearer on figure $2 \mathrm{c}$ the concentration of albumin-bound cortisol $\left(X_{F A}\right)$ is far from saturated, when cortisol is varied 0 to $2000 \mathrm{nM}$ for the default parameter values. Nguyen et al. [3] refer to the relationship between albumin and cortisol as non-saturable due to the high concentration of albumin and large dissociation constant [3]. As the ratio of the affinity constant and the concentration of albumin is assumed constant in the model of Coolens et al. [1], changing $X_{A 0}$ or $K_{F A}$ do not influence the result of their model (see figure $3 \mathrm{~b}$ and $3 \mathrm{~d}$ ). The effect of changing $X_{A 0}$ or $K_{F A}$ on the prediction of the model by Nguyen et al. [3] is similar to ours though the absolute values are different, while the effect on the predictions of Dorin et al. [2] is smaller.

The sensitivity analysis showed a very low sensitivity of the static model towards all parameters and input variable related to albumin $\left(X_{A 0}, K_{F A}\right.$, $\left.K_{P A}, K_{T A}\right)$. However, this does not mean that albumin is unimportant in the calculation of free cortisol. The sensitivity analysis was preformed with free cortisol as the model output. The decrease in free cortisol percentage is small (0.56 percentage points), when total albumin $\left(X_{A 0}\right)$ is varied from the minimum value $5.49 \cdot 10^{5} \mathrm{nM}$ to the maximum value $7.20 \cdot 10^{5} \mathrm{nM}$ of the normal range reported in Rustad et al. [46] (see figure 3) in good keeping with the sensitivity analysis. However, when looking at the distribution of cortisol on different forms in figure 3a cortisol bound to albumin simultaneously increases by 3.81 percentage points and cortisol bound to CBG on different forms decreases by 3.25 percentage points. Increasing the affinity of albumin for cortisol by varying $K_{F A}$ from the maximum to the minimum of the in the literature reported values have a larger effect by decreasing cortisol in the free form 3.81 percentage points, while CBG-bound forms decrease by 22.70 percentage points and albumin bound increase by 26.51 percentage points. 


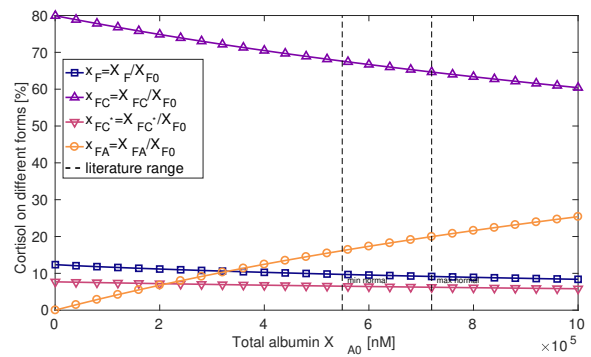

(a)

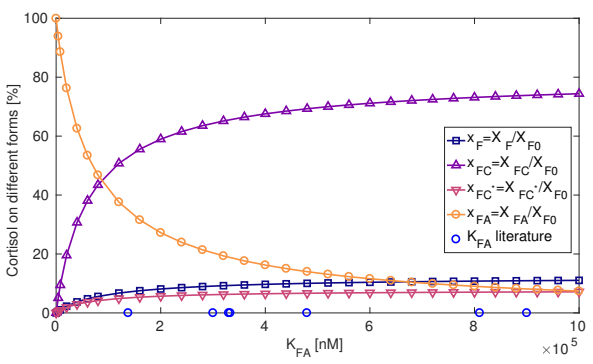

(c)

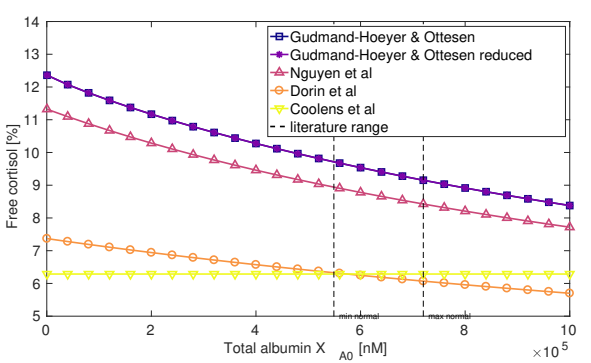

(b)

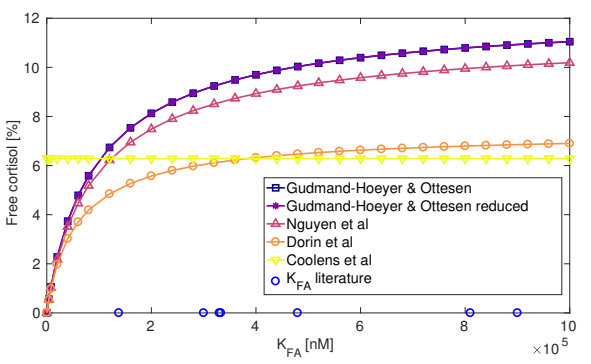

(d)

Figure 3: Varying total albumin and its dissociation constant for cortisol have great impact on redistribution of cortisol on bound forms. The total concentration of albumin $\left(X_{A 0}\right)$ is varied in a) and b) and the dissociation constant of albumin for cortisol $\left(K_{F A}\right)$ in c) and d). Minimum and maximum values for the normal range of albumin is indicated by vertical dotted lines $\left(5.49 \cdot 10^{5} \mathrm{nM}\right.$ to $\left.7.20 \cdot 10^{5} \mathrm{nM}[46]\right)$. The $K_{F A}$ values found in the literature (see table 1) is marked with circles on the x-axis. In a) and c) the calculated concentrations of cortisol in all forms as percentage of total cortisol is shown for the static model. In b) and d) the calculated concentrations of cortisol in the free form for the static model (Gudmand-Hoeyer \& Ottesen) and the reduced static model (Gudmand-Hoeyer \& Ottesen reduced) as well as the models in Nguyen et al. [3], Coolens et al. [1], and Dorin et al. [2] are shown as percentages of total cortisol. The reduced static and the static model show close to identical results. 


\subsubsection{The impact of $C B G$ variation}

As the sensitivity analysis in section 3.1 indicated, varying $X_{C 0}$ has a large impact on the level of free cortisol. A wide normal range such as the one reported in Lewis et al. [44] of 312 to $1324 \mathrm{nM}$ gives a 10.94 percentage points difference in by the static model estimated free cortisol percentage between maximum and minimum normal range values (see figure S.4 in Supplementary materials). Hence, the percentage of free cortisol could vary greatly from person to person and with the diurnal rhythm of CBG and cortisol combined in one person as well.

Common for all the models compared the percentage of free cortisol varies greatly between the smallest and the largest $K_{F C}$ reported in the literature, though the exact values are different with Nguyen et al. [3] and ours versus Coolens et al. [1] and Dorin et al. [2] being close in their predictions. The prediction for free cortisol for the static model changes 24.21 percentage points in this interval. The redistribution of cortisol bound to CBG or to albumin is even larger (see figure S.4 in Supplementary materials).

\subsubsection{Variation in the activity of elastase}

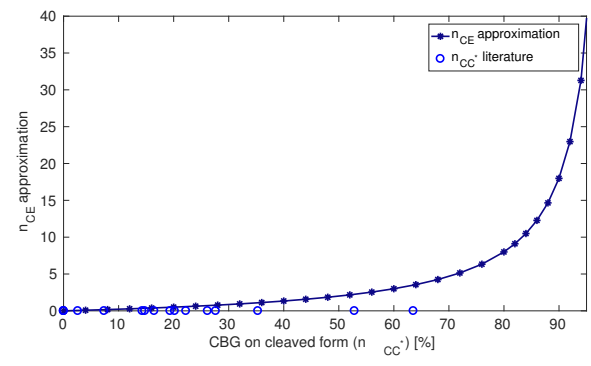

(a)

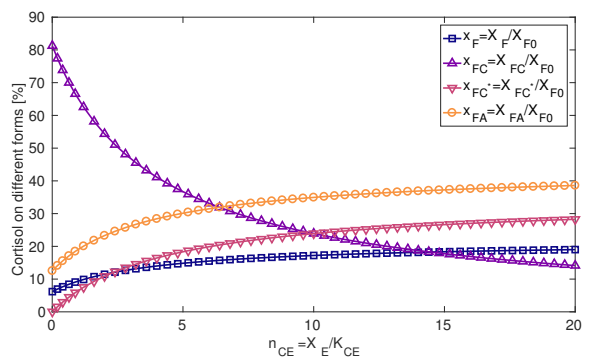

(b)

Figure 4: The cleavage of CBG by elastase has a great impact on cortisol distribution. a) The approximation of $n_{C E}$ as a function of how much of CBG is on cleaved form ( $n_{C E}=\frac{X_{E}}{K_{C E}} \approx \frac{k_{n c e} \cdot n_{C C^{*}}}{1-n_{C C^{*}}}$, with $\left.k_{n c e}=2\right)$. On the $\mathrm{x}$-axis $n_{C C^{*}}$-values for 16 normal subjects from Lewis and Elder [20] are indicated with circles. b) The fraction $n_{C E}=\frac{X_{E}}{K_{C E}}$ is varied. The calculated concentrations of cortisol in all forms as percentage of total cortisol given is shown for the static model.

Changing the fraction of CBG on elastase-cleaved form $\left(n_{C C^{*}}=\frac{C B G^{*} t o t}{X_{C 0}}\right)$ influence the model by Nguyen et al. [3] and through the approximation $n_{C E} \approx \frac{2 \cdot n_{C C^{*}}}{1-n_{C C^{*}}}$ the static and the reduced static model in a very similar way. 
The prediction by Coolens et al. [1] and Dorin et al. [2] are unaffected by change in $n_{C C^{*}}$, since the differentiation between CBG on cleaved and uncleaved form is not included in their models (see figure S.7 in Supplementary materials).

In figure 4a the approximation of $n_{C E}$ as a function of $n_{C C^{*}}$ is shown in the interval 0 to $95 \%$ to illustrate what happened to the approximated $n_{C E}$, when $n_{C C^{*}}$ is changed. The $n_{C C^{*}}$ for 16 normal subjects reported in Lewis and Elder [20] is indicated as circles on the x-axis. The approximated $n_{C E}$ values are similar for all 16 subjects including the two discordant samples compared to the drastic rise in approximated $n_{C E}$ values for higher $n_{C C^{*}}$ values.

In figure $4 \mathrm{~b}$ the parameter $n_{C E}$ is varied from 0 to 20 . As the figure shows, both models presented in this paper indicate that free cortisol concentration is very sensitive towards change in elastase activity.

The default value for the parameter $n_{C C^{*}}$ set to 0.325 gives with the approximation a default value of 0.963 for $n_{C E}$. In section 3.3 we investigate what happens, when we fit $n_{C E}$ individually to the data of four individuals with known levels of total cortisol, free cortisol, native CBG and cleaved CBG.

\subsubsection{The impact of testosterone and progesterone variation}

The steroid hormones testosterone and progesterone are included in our model as opposed to the three earlier models $[1,2,3]$. Hence, we are able to investigate their influences on free cortisol concentrations by varying the total concentrations $X_{T 0}$ and $X_{P 0}$ as well as their dissociation constants $K_{T C}$, $K_{T A}, K_{P C}$, and $K_{P A}$.

The effect of varying testosterone in the normal range for young men ( $\max 26.3 \mathrm{nM}[48]$ ) has an impact of $<0.03$ percentage points on cortisol on any form. Setting $X_{T 0}=26.3 n M$ the effect of varying $K_{T C}$ and $K_{T A}$ in the range of the in the literature found values are $<0.01$ percentage points in all forms of cortisol.

The effects of changing $K_{P C}$ and $K_{P A}$ are small ( $<0.1$ percentage points change of cortisol on any form, see figure S.10), when progesterone levels are at $85.9 \mathrm{nM}$ or less, but could have a higher impact for greater progesterone levels.

Figure 5 indicates that the fluctuations in progesterone levels seen during a normal menstrual cycle could affect free cortisol levels. A progesterone level of $85.9 \mathrm{nM}$, which is the maximum of the normal range in the luteal phase, 
increases the predicted free cortisol level by 0.14 percentage points. When the progesterone concentration increases some of the cortisol no longer bound to CBG binds to albumin instead (see figure 5). Including progesterone at the level seen in late pregnancy have a similar impact on cortisol distribution as changing albumin concentrations in the normal range, since free cortisol is increased by 0.85 percentage points, the fraction bound to albumin is increased by 1.70 percentage points, while the fraction bound to CBG is decreased by 2.55 percentage points.

However, these calculations are done by only changing the progesterone levels, while keeping all other parameters and input variables as their default values. In section 3.4 we will look at the predictions of the compared models on typical levels of all input variables for different population groups with pregnant women as one of these.

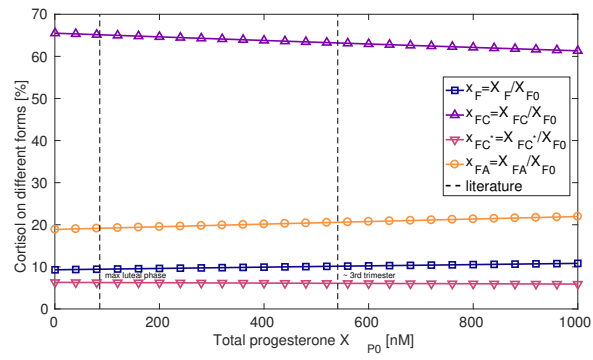

Figure 5: Progesterone at levels seen in the luteal phase and during pregnancy could influence the cortisol distribution. The total concentration of progesterone $\left(X_{P 0}\right)$ is varied. The maximum of the luteal phase (85.9 nM [8]) and the normal level of 3th trimester pregnancy (541 nM [12]) are marked with vertical dotted lines. The calculated concentrations of cortisol in all forms as percentage of total cortisol are shown for the static model.

\subsection{Different models' predictions for four individuals with measured free cor- tisol}

In table 3 estimates of free cortisol for four subjects described in [20] and [3] by the different methods stated in section 2.3 appear. The results are shown graphically in a bar diagram in figure 6 .

The approximated values for $\frac{X_{E}}{K_{C E}} \approx \frac{2 C_{t o t}^{*}}{C_{t o t}}$ in case $\mathrm{A}$ and $\mathrm{B}$ are 0.49 , 0.32, 2.18, and 3.99 for subject Concordant 1, Concordant 2, Discordant 1, and Discordant 2, respectively, while the corresponding individually fitted values in case $\mathrm{C}$ are $0.44,0.10,0.93$, and 0.84, respectively. Comparing the individually fitted $n_{C E}$ values with the approximated $\frac{X_{E}}{K_{C E}} \approx \frac{2 C_{t o t}^{*}}{C_{t o t}}$ for the 
four individual subjects, one see that for the concordant subjects the values are close, while they differ more for the discordant subjects.

With the individually fitted values for $n_{C E}$ in case $\mathrm{C}$ the model predictions are really close to data (see table 3 and figure 6 ). In case $\mathrm{D} n_{C E}$ is approximated from the level of cleaved CBG by $n_{C E} \approx \frac{k_{n c e} \cdot n_{C C^{*}}}{1-n_{C C^{*}}}$ and the factor $k_{n c e}$ is fitted as one common factor for all four subjects. The fitted value is $k_{n c e}=0.63$, i.e. the $n_{C E}$-values are $0.15,0.10,0.69$, and 1.26 for subject Concordant 1, Concordant 2, Discordant 1, and Discordant 2, respectively. The fit of our model in case D is not as perfect as with the individual fitted $n_{C E}$ in case C. However, our model with this common factor still performs better than any of the other models for three out of four subjects.

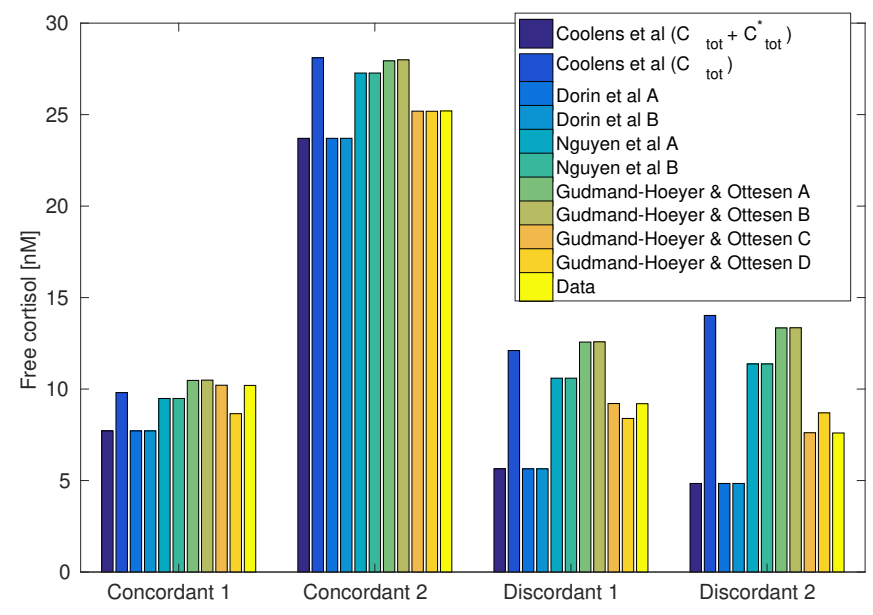

Figure 6: The model fits data excellently compared to previous models, when the elastase activity is fitted. Model predictions by Coolens et al. [1], Dorin et al. [2], Nguyen et al. [3] and our static model (Gudmand-Hoeyer \& Ottesen's formula) and data of free cortisol compared graphically for each of the four subjects Concordant 1, Concordant 2, Discordant 1 , and Discordant 2 described in [20]. Letters A, B, C, and D refer to the corresponding cases described in section 2.3. Measured input variables of [20] can be found in table 3 . The brackets after Coolens refer to whether the results were calculated on total CBG $\left(C_{t o t}+C_{t o t}^{*}\right)$ or only intact CBG $\left(C_{t o t}\right)$. 
Table 3: Measured and estimated free cortisol of four subjects described in [20].

\begin{tabular}{|l|r|r|r|r|}
\hline Sample & Concordant 1 & Concordant 2 & Discordant 1 & Discordant 2 \\
\hline$C_{\text {tot }}(\mathrm{nM})$ & 830 & 500 & 480 & 297 \\
\hline$C^{*}{ }_{t o t}(\mathrm{nM})$ & 204 & 79 & 524 & 592 \\
\hline$X_{F 0}$ & 217 & 307 & 162 & 127 \\
\hline$X_{F}(\mathrm{nM})$ & $\mathbf{1 0 . 2}$ & $\mathbf{2 5 . 2}$ & $\mathbf{9 . 2}$ & $\mathbf{7 . 6}$ \\
\hline$X_{F}(\%)$ & 4.70 & 8.21 & 5.68 & 5.98 \\
\hline Coolens formula: & & & & \\
On $C_{t o t}+C^{*}$ tot & 7.7 & 23.7 & 5.6 & 4.8 \\
On $C_{t o t}$ & 9.8 & 28.1 & 12.1 & 14.0 \\
\hline Dorin's formula: & & & & \\
Case A & 7.7 & 23.7 & 5.6 & 4.8 \\
Case B & 7.7 & 23.7 & 5.6 & 4.8 \\
\hline Nguyen's formula & & & & \\
Case A & 9.5 & 27.3 & 10.6 & 11.4 \\
Case B & 9.5 & 27.3 & 10.6 & 11.4 \\
\hline Gudmand-Hoeyer \& & & & & \\
Ottesen's formula & & & & \\
Case A & 10.5 & 27.9 & 12.6 & 13.3 \\
Case B & 10.5 & 28.0 & 12.6 & 13.4 \\
Case C & 10.2 & 25.2 & 9.2 & 7.6 \\
Case D & 8.7 & 25.2 & 8.4 & 8.7 \\
\hline
\end{tabular}

Measured native CBG concentration $\left(C_{t o t}\right)$, cleaved $\mathrm{CBG}^{*}$ concentration $\left(C^{*}{ }_{t o t}\right)$ and total and free cortisol concentrations $\left(X_{F 0}\right.$ and $\left.X_{F}\right)$ are shown for samples from four subjects Concordant 1, Concordant 2, Discordant 1, and Discordant 2 described in [20]. The results of different methods for calculating free cortisol are reported: Coolens et al. [1], Dorin et al. [2], Nguyen et al. [3] and our static model (Gudmand-Hoeyer \& Ottesen's formula). See a description of case A to $\mathrm{D}$ in section 2.3.

3.4. The impact of gender and age differences in albumin, testosterone and progesterone

Figure 7 shows the predicted distribution of cortisol in blood for estimated $24 \mathrm{~h}$ mean input variable values in different population groups made with the models by Coolens et al. [1] on total CBG (Coolens $\left(C_{t o t}+C_{t o t}^{*}\right)$ ), Coolens et al. [1] only including uncleaved CBG (Coolens $\left(C_{t o t}\right)$ ), Dorin et al. [2] (Dorin), Nguyen et al. [3] (Nguyen), our static model without the influence of progesterone and testosterone $(\mathrm{G} \& \mathrm{O}$ w/o P,T), our static model $(\mathrm{G}$ $\& \mathrm{O})$, and the reduced static model ( $\mathrm{G} \& \mathrm{O}$ reduced) for estimated $24 \mathrm{~h}$ averages of albumin, $\mathrm{CBG}, \mathrm{CBG}^{*}$, cortisol, progesterone and testosterone. The population groups are young men (7a), old men (7b), young women in 


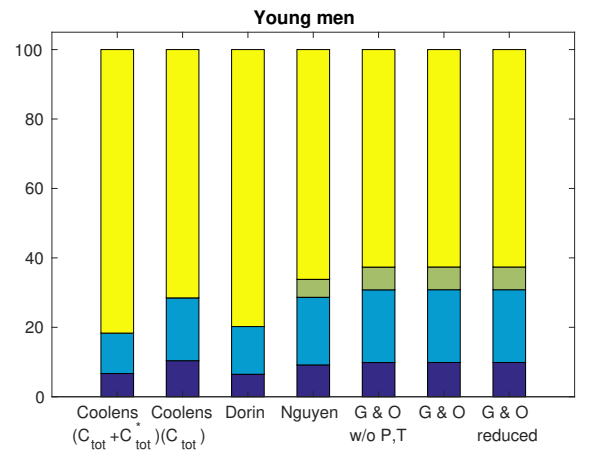

(a)

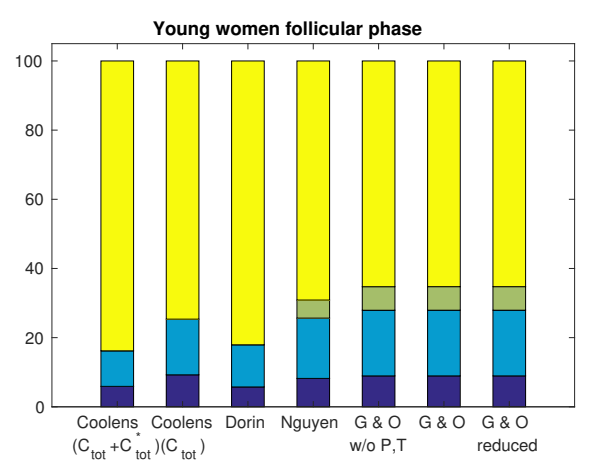

(c)

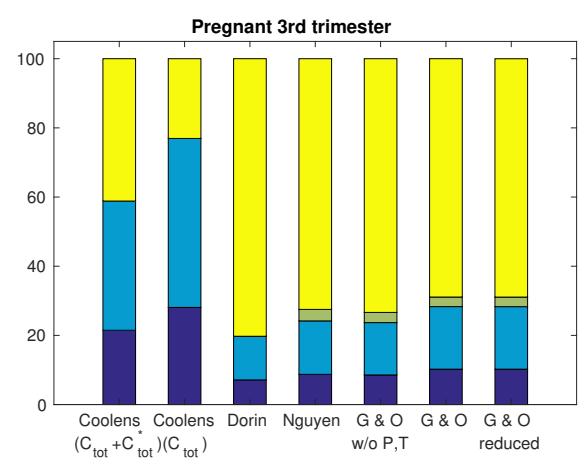

(e)

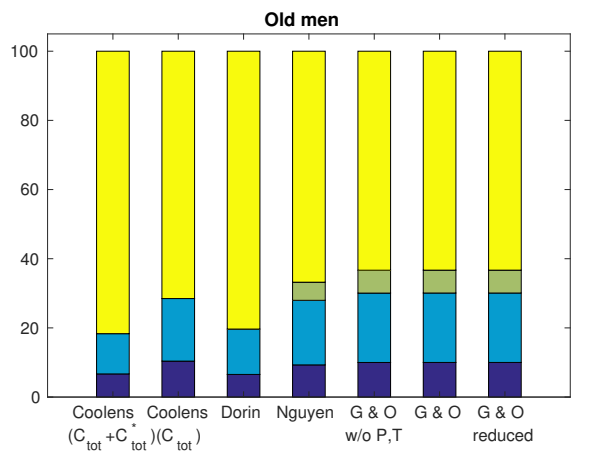

(b)

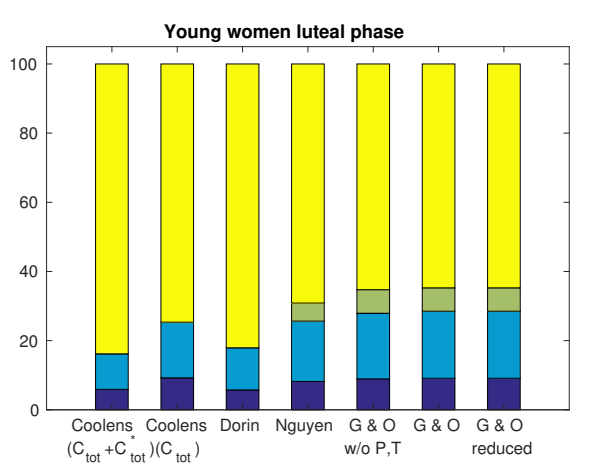

(d)

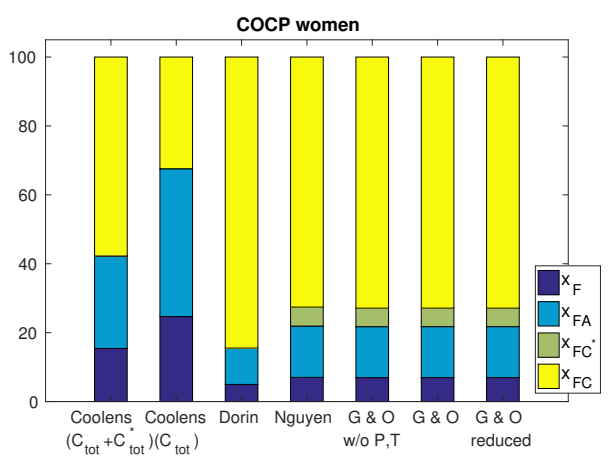

(f)

Figure 7: The model predictions of the models here and in [1], [2], and [3] differ between population groups. Estimates of $x_{F}$ (dark blue), $x_{F A}$ (light blue), $x_{F C^{*}}$ (green), and $x_{F C}$ (yellow) by Coolens et al. [1] on total CBG (Coolens $\left(C_{t o t}+C_{t o t}^{*}\right)$ ), Coolens et al. [1] on uncleaved CBG (Coolens $\left(C_{t o t}\right)$ ), Dorin et al. [2] (Dorin), Nguyen et al. [3] (Nguyen), the static model without progesterone and testosterone $(\mathrm{G} \& \mathrm{O}$ w/o $\mathrm{P}, \mathrm{T})$, the static model ( $\mathrm{G} \& \mathrm{O})$, and the reduced static model ( $\mathrm{G} \& \mathrm{O}$ reduced) for a) young men, b) old men, c) young women in the follicular phase, d) young women in the luteal phase, e) 3rd trimester pregnant women, and $\mathrm{f}$ ) women taking COGO (see table 2 for input variable values). The reduced static and the static model show close to identical results. 
their follicular phase (7c), young women in their luteal phase $(7 \mathrm{~d})$, pregnant women in 3rd trimester (7e), and women taking COCP (7f) (see table 2 for input variable values).

In general the differences between the models predictions for one of our six examples varies more than the individual models predictions for each of the six examples (see 7 a to $7 f$ ).

The influence of including progesterone and testosterone on the prediction of cortisol is small for both young and old men with cortisol on any form for both groups changing $<0.03$ percentage points when comparing the results of our model with and without progesterone and testosterone $(\mathrm{G} \& \mathrm{O}$ and $\mathrm{G}$ \& $\mathrm{O}$ reduced versus $\mathrm{G} \& \mathrm{O}$ w/o $\mathrm{P}, \mathrm{T})$. When comparing the predictions for young vs old men the difference in cortisol in the free form is approximately 0.14 percentage points for both the models by Dorin et al. [2], Nguyen et al. [3] and ours, while no difference is seen for the model by Coolens et al. [1]. This illustrate the effect of differences in albumin concentrations between young and old.

Even with the higher levels of total CBG and lower percentage of CBG being on cleaved form during pregnancy [60] the high level of progesterone clearly influences the distribution of cortisol on free and bound forms as seen when comparing the predictions of our static model with and without progesterone and testosterone $(\mathrm{G} \& \mathrm{O}$ and $\mathrm{G} \& \mathrm{O}$ reduced versus $\mathrm{G} \& \mathrm{O}$ $\mathrm{w} / \mathrm{o} \mathrm{P}, \mathrm{T})$ in figure $7 \mathrm{e}$. When progesterone and testosterone is included in the model, the percentage of cortisol in the free form $\left(x_{F}\right)$ is increased from $8.56 \%$ to $10.23 \%$ and bound to albumin $\left(x_{F A}\right)$ from $15.14 \%$ to $18.09 \%$ for our average pregnant woman. However, for women in their luteal phase an impact of progesterone can be seen too with an increase of 0.19 percentage points of cortisol being in the free form and 0.40 percentage points bound to albumin in the predictions of our model when compared to our model without progesterone and testosterone (see $\mathrm{G} \& \mathrm{O}$ and $\mathrm{G} \& \mathrm{O}$ reduced versus $\mathrm{G}$ \& $\mathrm{O}$ w/o P,T in figure $7 \mathrm{~d}$ ).

Women in 3rd trimester of pregnancy and women taking COCP (see figure 7 e and $7 f$, respectively) are both examples of circumstances where the women have high levels of CBG and total cortisol [60]. The difference between the predictions by the model by Coolens et al. [1] and the other models are greater for these two examples than for the remaining four. 


\section{Discussion}

In the present study we develop and validate a new static model for finding the concentration of free cortisol as well as determine the distribution of cortisol bound to albumin, intact and elastase cleaved CBG (CBG and CBG*, respectively). We suggest directly including elastase activity $n_{C E}=\frac{X_{E}}{K_{C E}}$ in the calculation of free cortisol with the approximated equilibrium dissociation constant $K_{C E}=\frac{k_{C^{*}}}{k_{C^{*}}^{+}} K_{M_{C E}}$ given by the Michaelis-Menten constant $\left(K_{M_{C E}}\right)$, the catalytic constant $\left(k_{C^{*}}^{+}\right)$, and the elimination constant $\left(k_{C^{*} e}\right)$ for the $\mathrm{CBG}^{*}$ synthesis and elimination.

If the level of elastase is unknown, but the level of $\mathrm{CBG}$ and $\mathrm{CBG}^{*}$ is know, one is able to use the approximation $n_{C E} \approx \frac{k_{n c e} C_{t o t}^{*}}{C_{t o t}}$. The results from fitting $k_{n c e}$ (or more generally $n_{n c e}$ ) individually to the data of four normal individuals in section 3.3 show that the model is able to fit data very well. The good performance of the model after fitting a common $k_{n c e}=0.63$ for all four subjects shows that the model can be used as an improved method for estimating free cortisol.

Additionally, by including the level of progesterone and testosterone in the model we are able to investigate the impact of these competitive steroids on cortisol distribution in the blood. A reduced version of the model is in the form of a fourth order polynomial and gives almost identical results as the static model for al investigations made (see section 3).

Even-though, there is a gender difference in the concentration of testosterone with normal young men having $17.7 \pm 1.0 n M[49]$ and normal premenopausal women having $1.4 \pm 0.2 n M$ [14] as well as an age-related change with elderly men having $12.1 \pm 0.7 n M$ [49], our model suggests that testosterone does not influence the free cortisol concentration significantly when varied in a physiologically relevant range. On top of this, testosterone in the blood binds to sex hormone binding globulin (SHBG) [27], which is not included in the model presented here, possibly further cancelling out the potential effect testosterone could have on the free cortisol concentration.

The affinity of human CBG for progesterone is of a considerable strength [81] with dissociation constants reported in the range of $11.1 \mathrm{nM}$ to $85 \mathrm{nM}$ [43, 7, 23, 81]. According to Cameron et al. [23] the binding of progesterone to both albumin and CBG do not influence the level of free cortisol significantly under physiological conditions despite the apparent contest between cortisol and progesterone in the binding to the transport proteins. Cameron et al. [23] attributes this to the relatively low concentration of progesterone 
relatively weak binding to both transport proteins [23]. Meyer et al. [81] argued that progesterone at high concentrations could become of importance in replacing cortisol [81]. Progesterone rises dramatically during pregnancy [12]. Meanwhile, both CBG and total cortisol rise as well with the rise in total cortisol probably being due to the rise in CBG [82]. Additionally, Nenke et al. [60] finds higher percentage of the uncleaved, high-affinity CBG in pregnant women and speculate that this could counteract the binding of progesterone [60]. Investigating these questions related to progesterone's impact, our simulations show that the static model predicts a rise in free cortisol not only when progesterone is varied independently as in section 3.2.5, but also when the parameters $X_{C 0}, X_{A 0}, X_{F 0}$, and $n_{C C^{*}}$ are set to typical values seen in pregnancy (see section 3.4). Moreover, changes in predicted free cortisol and redistribution of bound cortisol are seen already at levels corresponding to levels seen for women in the luteal phase of the menstrual cycle (see section 3.2.5 and 3.4). The changes in relation to including progesterone in the model is of the same magnitude as changes due to variation of albumin in the normal range (see section 3.2.2, 3.2.5, and 3.4).

Just as Dorin et al. [2] and Nguyen et al. [3] write in regard to their models, our static model can be used to estimate the affinities of cortisol to albumin and CBG. Dorin et al. [2] estimates the $K_{F A}$, but not $K_{F C}$, since they find that their model is not particular sensitive towards changes in $K_{F C}$. Our sensitivity analysis of the model in Dorin et al. [2], Nguyen et al. [3] and the models presented here shows greater sensitivity towards $K_{F C}$ than towards $K_{F A}$ when measured in absolute and relative sensitivity as well as a sensitivity measure normalized by the normal variation seen in the literature (see 3.1). Our sensitivity analysis and further investigations of varying the different input variables and parameters show that all the models are very dependent on the default parameter values when performing local sensitivity analysis and as a consequence local parameter estimations.

Both total and intact CBG has been shown to decrease with severity of sepsis, while $\mathrm{CBG}^{*}$ increased both measured in net concentration and percentage of total CBG [76]. The percentage of leucocytes circulating being neutrophils are in sepsis correlated with the relative and absolute levels of $\mathrm{CBG}^{*}[76]$. The model presented here is an equilibrium model describing the distribution of cortisol in plasma dependent on the activity of neutrophil elastase as measured in plasma. Korkmaz et al. [83] suggest neutrophil elastase as a therapeutic target [83]. By including the activity of neutrophil elastase directly in the model, we provide a method to investigate the influ- 
ence of such potential therapeutics on free cortisol concentrations in plasma. However, the lowering of the affinity of CBG for cortisol by cleavage by neutrophil elastase is thought to be a mechanism of local cortisol release in tissue with inflammation [84]. The concentration of neutrophil elastase release at localised inflammatory sites may far exceed the concentration measured in circulation. Hence, local relation between intact $\mathrm{CBG}$ and $\mathrm{CBG}^{*}$ in the interstitial compartments at sites of inflammation may differ greatly from the one measured in the circulation and, as a consequence, the level of free cortisol. The static model does not describe the concentration in the interstitial compartments, but describes the overall picture as seen in plasma. If a distinction between the levels seen in a high inflammatory interstitial compartment and the plasma compartment should be investigated, a dynamical model is needed.

In Nguyen et al. [3] a dynamic and spatially distributed model distinguishing between concentrations of cortisol and its transport proteins in the plasma and interstitial compartments is stated. Elastase is modelled only to cleave CBG in the interstitial compartment at locations with inflammation, while the synthesis of $\mathrm{CBG}^{*}$ and elimination of $\mathrm{CBG}$ in plasma is described independent of each other and by functions not directly related to the elastase activity, but dependent on the spatial location. Free cortisol is dramatically increased locally at inflammation sites due to the elastase activity in good agreement with the underlying biological understanding. However, when relating their dynamic model to their equilibrium formula Nguyen et al. [3] leave out the terms describing CBG-cleavage and metabolite concentration gradients.

To further validate the models presented in this article combined measurements of elastase activity, CBG and total and free cortisol are needed. As described in the section 2.2.3 the activity of neutrophil elastase can be measured by an activity based assay [66], immunologically using an antibody based assay directed towards neutrophil elastase [64, 70] or immunologically using an antibody based assay directed towards the complex NE- $\alpha_{1}$ AT $[67,68]$. However, these measurements may or may not be concordant. For example Kunder et al. [66] finds higher levels of neutrophil elastase activity in combination with lower levels of $\alpha_{1}$-AT and an absence of an increase in the levels of NE- $\alpha_{1} \mathrm{AT}$ in mild and severe preeclampsia [66]. Hence, measurements of NE- $\alpha_{1}$ AT alone would not have been a good biomarker of elastase activity in this case.

Furthermore, elastase might not be the only regulator of CBG on cleaved 
and uncleaved form. In a study by Nenke et al. [85] $\alpha_{1}$-AT deficient subjects, who lack this native neutrophil elastase inhibitor, paradoxically have higher levels of uncleaved CBG and lower levels of $\mathrm{CBG}^{*}$ [85].

Gender differences are present in the levels of the steroid hormones progesterone [8] and testosterone [9], but also in the levels of CBG [45]. The rise in cortisol and testosterone during pregnancy is often ascribed to the rise in the transport proteins CBG and sex hormone binding globulin (SHBG) [13]. However, the total concentration of cortisol is not solely controlled by the level of its transport proteins, since higher levels of CBG is seen in women compared to men [45], while higher levels of total cortisol is reported the same [86] or even higher [87] in men compared to women.

In subjects homozygote for a non-functioning CBG variant Perogamvros et al. [88] found similar free cortisol concentrations, but decreased glucocorticoid bioactivities when comparing these to subjects heterozygote for the CBG mutation and healthy controls [88]. When comparing the predictions of the different models for six example persons with estimated total levels of the input variables corresponding to different age and gender, it becomes clear that the models disagree on the distribution of cortisol in bound forms as well as their predictions for free cortisol (see figure 7). The most dramatic differences are seen in the predictions by Coolens et al. [1] for pregnant women and COCP women compared to the other models. Ho et al. [89] finds that the formulae by Coolens et al. [1] is not valid for calculating free cortisol in pregnant women [89]. Traditionally only free cortisol is considered bioactive and that the dissociations of cortisol from both albumin and CBG happen quite fast [90]. There has been speculation on a CBG receptor taking part in the activity of cortisol [91]. Hence, maybe not only the models predictions on free cortisol should be taken into consideration, but also the predictions on the distribution of bound cortisol.

Cortisol is secreted in ultradian pulses of approximately 1-2 hour period and with large amplitudes compared to their average values [92, 93]. By using a static equilibrium solution we assume that the equilibrium between the transport proteins and steroids occurs rapidly. This assumption is often applied in both methods for estimating free cortisol [2] and in dynamical models of the hypothalamic-pituitary-adrenal (hpa) axis [94]. Furthermore, by equation 1 we assume equilibrium between the elimination and enzymatic synthesis of $\mathrm{CBG}^{*}$. As discussed above the activity of elastase could differ greatly between the blood and sites of inflammation. The dynamical model by Nguyen et al. [3] is in the time scale of seconds, but they do not relate their 
work to the ultradian and circadian oscillations present in the system. In an ongoing work we are exploring a dynamical version of the underlying mass action model to see whether these assumptions hold and how the interaction of cortisol with plasma proteins in the blood influences the oscillating nature of cortisol.

\section{Acknowledgements}

We would like to thank Prof. Dr Richard I. Dorin for helpful discussions on parameter values. This research was supported by funding from The

Danish Society for the Protection of Laboratory Animals and from Alternativfondet.

\section{Conflict of interest disclosure}

The authors report no potential conflicts of interest. 


\section{Appendix A. Polynomial coefficients}

The polynomial coefficients in equation (9a) are,

$$
\begin{aligned}
a_{0}= & p_{0} p_{F C} p_{T C} p_{P C} \\
a_{1}= & p_{0} p_{F C} p_{T C}+p_{0} p_{T C} p_{P C}-p_{F C} p_{T C} p_{P C}+p_{0} p_{F C} p_{P C} \\
& +p_{C F} p_{T C} p_{P C}+p_{C P} p_{F C} p_{T C}+p_{0} p_{F A} p_{T C} p_{P C} \\
& +p_{C T} p_{F C} p_{P C}+p_{0} p_{F C} p_{T C} p_{P A}+p_{0} p_{F C} p_{T A} p_{P C} \\
a_{2}= & p_{C P} p_{F C} p_{T A}+p_{C P} p_{F A} p_{T C}+p_{0} p_{F C} p_{T A}+p_{0} p_{F A} p_{T C} \\
& -p_{T C} p_{P C}+p_{0} p_{P C}+p_{C T} p_{P C}+p_{C F} p_{P C}-p_{F C} p_{P C} \\
& +p_{0} p_{T C}+p_{0} p_{F C}+p_{C F} p_{T C}+p_{C T} p_{F C}-p_{F C} p_{T C} \\
& +p_{C P} p_{T C}+p_{C P} p_{F C}+p_{C F} p_{T C} p_{P A}+p_{0} p_{T A} p_{P C} \\
& -p_{F C} p_{T A} p_{P C}+p_{0} p_{T C} p_{P A}+p_{0} p_{F C} p_{P A}+p_{0} p_{F A} p_{P C} \\
& +p_{C T} p_{F C} p_{P A}+p_{C T} p_{F A} p_{P C}-p_{F C} p_{T C} p_{P A}-p_{F A} p_{T C} p_{P C} \\
& +p_{C F} p_{T A} p_{P C}+p_{0} p_{F A} p_{T A} p_{P C}+p_{0} p_{F C} p_{T A} p_{P A} \\
& +p_{0} p_{F A} p_{T C} p_{P A} \\
a_{3}= & p_{C P} p_{F A} p_{T A}+p_{0} p_{F A} p_{T A}-p_{F C}+p_{C P}+p_{C T}+p_{C F} \\
& +p_{0}-p_{P C}-p_{T C}-p_{T C} p_{P A}-p_{T A} p_{P C}+p_{0} p_{P A}+p_{C T} p_{P A} \\
& +p_{C F} p_{P A}-p_{F A} p_{P C}-p_{F C} p_{P A}+p_{0} p_{T A}+p_{0} p_{F A} \\
& +p_{C F} p_{T A}+p_{C T} p_{F A}-p_{F C} p_{T A}-p_{F A} p_{T C}+p_{C P} p_{T A} \\
& +p_{C P} p_{F A}-p_{F C} p_{T A} p_{P A}-p_{F A} p_{T A} p_{P C}-p_{F A} p_{T C} p_{P A} \\
& +p_{C T} p_{F A} p_{P A}+p_{0} p_{T A} p_{P A}+p_{C F} p_{T A} p_{P A}+p_{0} p_{F A} p_{P A} \\
& +p_{0} p_{F A} p_{T A} p_{P A} \\
a_{4}= & -1-p_{F A} p_{T A}-p_{T A} p_{P A}-p_{F A} p_{P A} \\
& -p_{P A}-p_{T A}-p_{F A}-p_{F A} p_{T A} p_{P A}
\end{aligned}
$$




\section{Appendix B. Solution formulae for the reduced static model}

Several methods exist to, by help of radicals, finding analytical solutions to fourth order polynomials (also referred to as "quartics") [95]. We use the basic algorithm stated in Shmakov [95] and find the polynomial for the parameter values investigated to have four real roots of which one is positive and three are negative. Hence, the following positive root is the only physiological relevant solution to the model:

$$
x_{C}=\frac{-g+\sqrt{g^{2}-4 \cdot h}}{2}
$$

where

$$
\begin{aligned}
& g=\frac{\frac{a_{1}}{a_{0}}-\sqrt{\left(\frac{a_{1}}{a_{0}}\right)^{2}-4 \cdot\left(\frac{a_{2}}{a_{0}}-y_{s}\right)}}{2} \\
& h=\frac{y_{s}-\sqrt{y_{s}^{2}-4 \cdot \frac{a_{4}}{a_{0}}}}{2}
\end{aligned}
$$

$y_{s}$ is a real root for the cubic equation $y^{3}+b \cdot y^{2}+c \cdot y+d=0$, with

$$
\begin{aligned}
b & =-\frac{a_{2}}{a_{0}}, \\
c & =\frac{a_{1}}{a_{0}} \cdot \frac{a_{3}}{a_{0}}-4 \cdot \frac{a_{4}}{a_{0}}, \\
d & =-\left(\frac{a_{1}}{a_{0}}\right)^{2} \cdot \frac{a_{4}}{a_{0}}-\left(\frac{a_{3}}{a_{0}}\right)^{2}+4 \cdot \frac{a_{2}}{a_{0}} \cdot \frac{a_{4}}{a_{0}} .
\end{aligned}
$$

$y_{s}$ can be found following the Tschirnhaus-Vieta approach [96]:

$$
y_{s}=A \cdot \cos \left(\frac{1}{3} \cdot \phi\right)+B
$$


where

$$
\begin{aligned}
& A=2 \cdot \sqrt{\frac{-p}{3}} \\
& \phi=\cos ^{-1}\left(\frac{3 \cdot q}{A \cdot p}\right) \\
& B=\frac{-b}{3}, \\
& p=\frac{3 \cdot c-b^{2}}{3}, \text { and } \\
& q=\frac{2 \cdot b^{3}-9 \cdot b \cdot c+27 \cdot d}{27}
\end{aligned}
$$




\section{References}

[1] J.-L. Coolens, H. van Baelen, W. Heyns, Clinical use of unbound plasma cortisol as calculated from total cortisol and corticosteroid-binding globulin, J. Steroid Biochem. 26 (2) (1987) 197-202.

[2] R. I. Dorin, H. K. Pai, J. T. Ho, J. G. Lewis, D. J. Torpy, F. K. Urban, C. R. Qualls, Validation of a simple method of estimating plasma free cortisol: role of cortisol binding to albumin, Clin. Biochem. 42 (2009) 64-71.

[3] P. T. T. Nguyen, J. G. Lewis, J. Sneyd, R. S. F. Lee, D. J. Torpy, P. R. Shorten, Development of a formula for estimating plasma free cortisol concentration from a measured total cortisol concentration when elastase-cleaved and intact corticosteroid binding globulin coexist, J. Steroid Biochem. Mol. Biol. 141 (2014) 16-25.

[4] C. J. Pretorius, J. P. Galligan, B. C. McWhinney, S. E. Briscoe, J. P. Ungerer, Free cortisol method comparison: ultrafiltation, equilibrium dialysis, tracer dilution, tandem mass spectrometry and calculated free cortisol, Clin. Chim. Acta 412 (11) (2011) 1043-1047.

[5] F. Kirchhoff, J. Briegel, M. Vogeser, Quantification of free serum cortisol based on equilibrium dialysis and isotope dilution-liquid chromatography-tandem mass spectrometry, Clin. Biochem. 44 (10) (2011) 894-899.

[6] N. El-Farhan, D. A. Rees, C. Evans, Measuring cortisol in serum, urine and saliva - are our assays good enough?, Ann. Clin. Biochem. 54 (3) (2017) 308-322.

[7] J. Dunn, B. Nisula, D. Rodbard, Transport of steroid hormones: binding of 21 endogenous steroids to both testosterone-binding globulin and corticosteroid-binding globulin in human plasma, J. Clin. Endocrinol. Metab. 53 (1) (1981) 58-68.

[8] NIH Clinical Center, Test Guide Historical reference range Progesterone, NIH Clinical Center, URL http://cclnprod.cc.nih. gov/dlm/testguide.nsf/0/CB26894E1EB28DEF85256BA5005B000E? OpenDocument, Accessed on September 28th 2015, 2015. 
[9] NIH Clinical Center, Test Guide Total Testosterone, URL http://cclnprod.cc.nih.gov/dlm/testguide.nsf/Index/ 205A72FED5656E4285256B990064B432?OpenDocument, Accessed on September 28th 2015, 2015.

[10] J. Roos, S. Johnson, S. Weddell, E. Godehardt, J. Schiffner, G. Freundl, C. Gnoth, Monitoring the menstrual cycle: Comparison of urinary and serum reproductive hormones referenced to true ovulation, Eur. J. Contracept. Reproductive Health Care Early Online (2015) 1-13, doi: 10.3109/13625187.2015.1048331.

[11] A. Salonia, M. Pontillo, R. Nappi, G. Zanni, F. Fabbri, M. Scavini, R. Daverio, A. Gallina, P. Rigatti, E. Bosi, P. A. Bonini, F. Montorsi, Menstrual Cycle-Related Changes in Circulating Androgens in Healthy Women With Self-Reported Normal Sexual Function, J. Sex. Med 5 (2008) 854-863, doi: 10.1111/j.1743-6109.2008.00791.x.

[12] P. M. Meulenberg, J. A. Hofman, Salivary progesterone excellently reflects free and total progesterone in plasma during pregnancy, Clin. Chem. 35 (1) (1989) 168-172.

[13] M. H. Laudat, B. Guilhaume, P. Blot, C. Fournier, J. P. Giauque, J. P. Luton, État hormonal de la grossesse: modification du cortisol et de la testostérone, Annales d'endocrinologie (Paris) 48 (1987) 334-338.

[14] C. W. Bardin, M. B. Lipsett, Testosterone and androstenedione blood production rates in normal women and women with idiopathic hirsutism or polycystic ovaries, J. Clin. Investig. 46 (5) (1967) 891-902.

[15] D. E. Henley, S. L. Lightman, New insights into corticosteroid-binding globulin and glucocorticoid delivery, Neuroscience 180 (2011) 1-8.

[16] W. Rosner, The function of corticosteroid-binding globulin and sex hormone-binding globulin: Recent advances, Endocr. Rev. 11 (1) (1990) 80-91.

[17] J. G. Lewis, K. Saunders, A. Dyer, P. A. Elder, The half-lives of intact and elastase cleaved human corticosteroid-binding globulin (CBG) are identical in the rabbit, J. Steroid Biochem. Mol. Biol. 149 (2015) 53-57. 
[18] M. Simard, L. A. Hill, C. M. Underhill, B. O. Keller, I. Villanueva, R. E. W. Hancock, H. GL., Pseudomonas aeruginosa elastase disrupts the cortisol-binding activity of Cortisosteroid-binding glubelin, Endocrinology 155 (8) (2014) 2900-2908.

[19] W. L. Chan, R. W. Carrell, A. Zhou, R. J. Read, How changes in affinity of corticosteroid-binding globulin modulate free cortisol concentration, J. Clin. Endocrinol. Metab. 98 (8) (2013) 3315-3322.

[20] J. G. Lewis, P. A. Elder, Corticosteroid-binding globulin reactive centre loop antibodies recognise only the intact natured protein: Elastase cleaved and uncleaved CBG may coexist in circulation, J. Steroid Biochem. Mol. Biol. 127 (3) (2011) 289-294.

[21] H.-Y. Lin, C. Underhill, B. R. Gardill, M. YA., G. L. Hammond, Residues in the human corticosteroid-binding globulin reactive center loop that influence steroid binding before and after elastase cleavage, J. Biol. Chem. 284 (2) (2009) 884-896.

[22] J. L. Malisch, D. G. Satterlee, J. F. Cockrem, H. Wada, C. W. Breuner, How acute is the acute stress response? Baseline corticosterone and corticosteroid-binding globulin levels change $24 \mathrm{~h}$ after an acute stressor in Japanese quail, General and comparative endocrinology 165 (2) (2010) 345-350.

[23] A. Cameron, D. Henley, R. Carrell, A. Zhou, A. Clarke, S. Lightman, Temperature-responsive release of cortisol from its binding globulin: a protein thermocouple, J. Clin. Endocrinol. Metab. 95 (10) (2010) 46894695 .

[24] P. A. Pemberton, P. E. Stein, M. B. Pepys, J. M. Potter, R. W. Carrell, Hormone binding globulins undergo serpin conformational change in inflammation, Nature 336 (17) (1988) 257-257.

[25] L. L. Klosterman, J. T. Murai, P. K. Siiteri, Cortisol levels, binding, and properties of corticosteroid-binding globulin in the serum of primates, Endocrinology 118 (1) (1986) 424-434.

[26] M. M. Pugeat, J. F. Dunn, B. C. Nisula, Transport of steroid hormones: interaction of 70 drugs with testosterone-binding globulin and 
corticosteroid-binding globulin in human plasma, J. Clin. Endocrinol. Metab. 53 (1) (1981) 69-75.

[27] N. Mazer, A novel spreadsheet method for calculating the free serum concentrations of testosterone, dihydrotestosterone, estradiol, estrone and cortisol: with illustrative examples from male and female populations, Steroids 74 (2009) 512-519.

[28] M. M. Pugeat, G. P. Chrousos, B. C. Nisula, D. L. Loriaux, D. Brandon, M. B. Lipsett, Plasma cortisol transport and primate evolution, Endocrinology 115 (1) (1984) 357-361.

[29] J. F. Tait, S. A. S. Tait, The effect of plasma protein binding on the metabolism of steroid hormones, J. Endocrinol. 131 (3) (1991) 339-357.

[30] K. E. Mickelson, J. Forsthoefel, U. Westphal, Steroid-protein interactions. Human corticosteroid binding globulin: some physicochemical properties and binding specificity, Biochemistry 20 (21) (1981) 62116218.

[31] S. D. Stroupe, G. B. Harding, M. W. Forsthoefel, U. Westphal, Kinetic and equilibrium studies on steroid interaction with human corticosteroid binding globulin, Biochemistry 17 (1) (1978) 177-182.

[32] U. Westphal, Steroid Protein Interactions, Springer-Verlag, New York, 1971.

[33] V. Gayrard, M. Alvinerie, P. L. Toutain, Interspecies variations of corticosteroid-binding globulin parameters, Domest. Anim. Endocrinol. 13 (1) (1996) 35-45.

[34] L. I. McKlay, J. A. Cidlowski, Corticosteroids, Pharmacokinetics of Corticosteroids, in: D. W. Kufe, R. E. Pollock, R. R. Weichselbaum, R. C. Bast, T. S. Gansler, J. F. Holland, E. Frei (Eds.), HollandFrei Cancer Medicine, chap. 62, Hamilton (ON): BC Decker, 6 edn., URL https://www.ncbi.nlm.nih.gov/books/NBK13300, Accessed on December 4th 2017, 2003.

[35] X. Qi, F. Loiseau, W. L. Chan, Y. Yan, Z. Wei, L.-G. Milroy, R. M. Myers, S. V. Ley, R. J. Read, R. W. Carrell, et al., Allosteric modulation 
of hormone release from thyroxine and corticosteroid-binding globulins, J. Biol. Chem. 286 (18) (2011) 16163-16173.

[36] G. McCarty, B. Schwartz, Reduced plasma cortisol binding to albumin in ocular hypertension and primary open-angle glaucoma, Curr Eye Res. 18(6) (1999) 467-476.

[37] R. Södergård, T. Bäckström, V. Shanbhag, H. Carstensen, Calculation of free and bound fractions of testosterone and estradiol-17 $\beta$ to human plasma proteins at body temperature, J. Steroid Biochem. 16 (6) (1982) 801-810.

[38] D. C. Anderson, Sex-hormone-binding globulin, Clin. Endocrinol. 3 (1) (1974) 69-96.

[39] J. Moll, R. Rosenfield, J. Helke, Estradiol-testosterone binding interactions and free plasma estradiol under physiological conditions, J. Clin. Endocrinol. Metab. 52(5) (1981) 868-874.

[40] A. Vermeulen, The physical state of testosterone in plasma, in: S. M. James VHT, L. Martini (Eds.), The endocrine function of the human testis, vol. 1, Academic Press, New York, 157-170, 1973.

[41] X. Zheng, C. Bi, M. Brooks, D. S. Hage, Analysis of hormoneprotein binding in solution by ultrafast affinity extraction: interactions of testosterone with human serum albumin and sex hormone binding globulin, Anal. Chem. 87 (22) (2015) 11187-11194, doi: 10.1021/acs.analchem.5b03007.

[42] A. F. Clark, C. E. Bird, Binding of 5-androstane-3,17-diol to human plasma proteins, J. Endocrinol. 57(2) (1973) 289-298.

[43] U. Westphal, Steroid-protein interactions. XIII. Concentrationand binding affinities of corticosteroid-binding globulins in sera of man, monkey, rat, rabbit, and guinea pig, Ach. Biochem. 18 (3) (1967) 556-567.

[44] J. G. Lewis, C. J. Bagley, P. A. Elder, A. W. Bachmann, D. J. Torpy, Plasma free cortisol fraction reflects levels of functioning corticosteroid-binding globulin, Clin. Chim. Acta 359 (2005) 189-194, doi:10.1016/j.cccn.2005.03.044. 
[45] J. G. Lewis, P. A. Elder, Intact or "active" corticosteroid-binding globulin (CBG) and total CBG in plasma: Determination by parallel ELISAs using monoclonal antibodies, Clin. Chim. Acta 416 (2013) 26-30.

[46] P. Rustad, P. Felding, L. Franzson, V. Kairisto, A. Lahti, A. Mårtensson, P. H. Petersen, P. Simonsson, H. Steensland, A. Uldall, The Nordic Reference Interval Project 2000: recommended reference intervals for 25 common biochemical properties, Scand. J. Clin. Lab. Invest. 64 (4) (2004) 271-284, doi: 10.1080/00365510410006324.

[47] J. Helmersson-Karlqvist, P. Ridefelt, L. Lind, A. Larsson, Reference values for 34 frequently used laboratory tests in 80-year-old men and women, Maturitas 92 (2016) 97-101.

[48] Beckman Coulter Inc., Access Testosterone, URL https: //www . beckmancoulter. com/wsrportal/bibliography?docname= DS12798A\%20Access $\% 20$ Testosterone $\% 20 W W \% 20$ Data $\% 20$ Sheet.pdf, Accessed on December 4th 2017, 2009.

[49] S. R. Plymate, J. S. Tenover, W. J. Bremner, Circadian Variation in Testosterone, Sex Hormone-Binding Globulin, and Calculated Non-Sex Hormone-Binding Globulin Bound Testosterone in Healthy Young and Elderly Men, J. Androl. 10 (5) (1989) 366-371.

[50] X. Shen, R. Wang, N. Yu, Y. Shi, H. Li, C. Xiong, Y. Li, E. M. Wells, Y. Zhou, Reference Ranges and Association of Age and Lifestyle Characteristics with Testosterone, Sex Hormone Binding Globulin, and Luteinizing Hormone among 1166 Western Chinese Men, PloS one 11 (10) (2016) e0164116.

[51] M. Le, D. Flores, D. May, E. Gourley, A. K. Nangia, Current practices of measuring and reference range reporting of free and total testosterone in the United States, J. Urol. 195 (5) (2016) 1556-1561.

[52] A. L. Southren, S. Tochimoto, N. C. Carmody, K. Isurugi, Plasma Production Rates of Testosterone in Normal Adult Men and Women and in Patients with the Syndrome of Feminizing Testes 1, J. Clin. Endocrinol. Metab. 25 (11) (1965) 1441-1450.

[53] A. L. Southren, G. G. Gordon, S. Tochimoto, G. Pinzon, D. R. Lane, W. Stypulkowski, Mean plasma concentration, metabolic clearance and 
basal plasma production rates of testosterone in normal young men and women using a constant infusion procedure: effect of time of day and plasma concentration on the metabolic clearance rate of testosterone, J. Clin. Endocrinol. Metab. 27 (5) (1967) 686-694.

[54] H. Junkermann, H. Mangold, P. Vecsei, B. Runnebaum, Circadian rhythm of serum progesterone levels in human pregnancy and its relation to the rhythm of cortisol, Acta Endocrinol. 101 (1) (1982) 98-104.

[55] T. F. Gallagher, K. Yoshida, H. D. Roffwarg, D. K. Fukushima, E. D. Weitzman, L. Hellman, ACTH and Cortiol Secretory Patterns in Man, J. Clin. Endocrinol. Metab. 36 (1973) 1058-1068.

[56] E. Aardal, A.-C. Holm, Cortisol in saliva-reference ranges and relation to cortisol in serum, Clin. Chem. Lab. Med. 33 (12) (1995) 927-932.

[57] Roche, Cortisol (serum, plasma), URL http://www.acb.org.uk/Nat\% 20Lab\%20Med\%20Hbk/Cortisol.pdf, Accessed on December 4th 2017, 2012.

[58] B. Allolio, J. Hoffmann, E. Linton, W. Winkelmann, M. Kusche, H. M. Schulte, Diurnal salivary cortisol patterns during pregnancy and after delivery: relationship to plasma corticotrophin-releasing-hormone, Clin. Endocrinol. 33 (2) (1990) 279-289.

[59] F. Murao, A. Yasuda, T. Shibukawa, K. Takahashi, K. Sawada, K. Kaneda, K. Hasegawa, M. Kitao, Human corticosteroid-binding capacities in normal, high-risk or pregnancies with an abnormal outcome, Acta Obst Gynaec Jpn 38 (4) (1986) 590-594.

[60] M. A. Nenke, A. Zeng, E. J. Meyer, J. G. Lewis, W. Rankin, J. Johnston, S. Kireta, S. Jesudason, D. J. Torpy, Differential Effects of Estrogen on Corticosteroid-Binding Globulin Forms Suggests Reduced Cleavage in Pregnancy, J. Endocrine Society 1 (3) (2017) 202-210, doi:10.1210/js.2016-1094.

[61] Y. Bacq, O. Zarka, J. F. Bréchot, N. Mariotte, S. Vol, J. Tichet, J. Weill, Liver function tests in normal pregnancy: a prospective study of 103 pregnant women and 103 matched controls, Hepatology 23 (5) (1996) 1030-1034. 
[62] A. Larsson, M. Palm, L.-O. Hansson, O. Axelsson, Reference values for clinical chemistry tests during normal pregnancy, BJOG 115 (7) (2008) 874-881, doi: 10.1111/j.1471-0528.2008.01709.x.

[63] C. Kirschbaum, B. M. Kudielka, J. Gaab, N. C. Schommer, D. H. Hellhammer, Impact of gender, menstrual cycle phase, and oral contraceptives on the activity of the hypothalamus-pituitary-adrenal axis, Psychosom. Med. 61 (1999) 154-162.

[64] T. Kodama, H. Yukioka, T. Kato, N. Kato, F. Hato, S. Kitagawa, Neutrophil elastase as a predicting factor for development of acute lung injury, Intern. Med. 46 (11) (2007) 699-704.

[65] J. J. Sng, S. Prazakova, P. S. Thomas, C. Herbert, MMP-8, MMP-9 and Neutrophil Elastase in Peripheral Blood and Exhaled Breath Condensate in COPD, COPD: Journal of Chronic Obstructive Pulmonary Disease 14 (2) (2017) 238-244.

[66] M. Kunder, A. V. M. Kutty, V. Lakshmaiah, S. R. Sheela, Correlation of Plasma Neutrophil Elastase Activity and Endogenous Protease Inhibitor Levels with the Severity of Pre-eclampsia, J. Clin. Diagn. Res. 11 (3) (2017) BC09-BC12.

[67] B. Polańska, A. Turno-Kręcicka, M. Kaźmierowska-Niemczuk, Assessment of neutrophil elastase plasma levels in children with intermediate uveitis, Cent. Eur. J. Immunol. 37 (3) (2012) 275-279.

[68] D. Pawlica-Gosiewska, B. Solnica, K. Gawlik, D. Cibor, T. Mach, D. Fedak, D. Owczarek, The use of selected neutrophil protein plasma concentrations in the diagnosis of Crohn's disease and ulcerative colitisa preliminary report, Postepy. Hig. Med. Dosw. (online), 71 (2017) 243.

[69] M. Zbikowska-Gotz, K. Palgan, E. Socha, M. Przybyszewski, A. Kuzminski, Z. Bartuzi, Measurement of effector properties of neutrophilic granulocytes in patients with allergic hypersensitivity to food, Post. Dermatol. Alergol. 28 (3) (2011) 175.

[70] S. C. Donnelly, I. MacGregor, A. Zamani, M. Gordon, C. E. Robertson, D. J. Steedman, K. Little, C. Haslett, Plasma elastase levels and the development of the adult respiratory distress syndrome, Am. J. Respir. Crit. Care Med. 151 (5) (1995) 1428-1433. 
[71] A. A. Sandberg, M. Woodruff, H. Rosenthal, S. Nienhouse, W. Slaunwhite Jr, Transcortin: a corticosteroid-binding protein of plasma. VII. Half-life in normal and estrogen-treated subjects, J. Clin. Invest. 43 (3) (1964) 461.

[72] Information on EC 3.4.21.37 - leukocyte elastase, URL https:// www . brenda-enzymes . org/enzyme . php?ecno=3.4.21.37, Accessed on September 25th 2017, 2017.

[73] K. Nakajima, J. C. Powers, B. M. Ashe, M. Zimmerman, Mapping the extended substrate binding site of cathepsin $G$ and human leukocyte elastase. Studies with peptide substrates related to the alpha 1-protease inhibitor reactive site., J. Biol. Chem. 254 (10) (1979) 4027-4032.

[74] G. L. Hammond, C. L. Smith, N. A. M. Paterson, W. J. Sibbald, A role for corticosteroid-binding globulin in delivery of cortisol to activated neutrophils, J. Clin. Endocrinol. Metab. 71 (1) (1990) 34-39.

[75] Z. Sumer-Bayraktar, O. C. Grant, V. Venkatakrishnan, R. J. Woods, N. H. Packer, M. Thaysen-Andersen, Asn ${ }^{347}$ glycosylation of corticosteroid-binding globulin fine-tunes the host immune response by modulating proteolysis by Pseudomonas aeruginosa and neutrophil elastase, J. Biol. Chem. 291 (34) (2016) 17727-17742.

[76] M. A. Nenke, W. Rankin, M. J. Chapman, N. E. Stevens, K. R. Diener, J. D. Hayball, J. G. Lewis, D. J. Torpy, Depletion of high-affinity corticosteroid-binding globulin corresponds to illness severity in sepsis and septic shock; clinical implications, Clin. Endocrinol. 82 (6) (2015) 801-807, doi: 10.1111/cen.12680.

[77] B. M. Kudielka, A. Buske-Kirschbaum, D. H. Hellhammer, C. Kirschbaum, HPA axis responses to laboratory psychosocial stress in healthy elderly adults, younger adults, and children: impact of age and gender, Psychoneuroendocrinology 29 (1) (2004) 83-98.

[78] K. Okazaki, H. Hayase, T. Ichinose, H. Mitono, T. Doi, H. Nose, Protein and carbohydrate supplementation after exercise increases plasma volume and albumin content in older and young men, J. Appl. Physiol. 107 (3) (2009) 770-779, doi:10.1152/japplphysiol.91264.2008. 
[79] J. D. Peck, B. S. Hulka, C. Poole, D. A. Savitz, D. Baird, B. E. Richardson, Steroid hormone levels during pregnancy and incidence of maternal breast cancer, Cancer Epidemiol. Biomarkers Prev. 11 (4) (2002) 361368.

[80] Roche, Testosterone II, URL http://objednavky. roche-diagnostics.cz/download/metody/Detailni_informace o_metode.pdf, accessed on August 21th 2017, 2010.

[81] E. J. Meyer, M. A. Nenke, W. Rankin, J. G. Lewis, D. J. Torpy, Corticosteroid-binding globulin: a review of basic and clinical advances, Horm. Metab. Res. 48 (06) (2016) 359-371.

[82] E. Demey-Ponsart, J.-M. Foidart, J. Sulon, J. C. Sodoyez, Serum CBG, free and total cortisol and circadian patterns of adrenal function in normal pregnancy, J. Steroid Biochem. 16 (2) (1982) 165-169.

[83] B. Korkmaz, M. S. Horwitz, D. E. Jenne, F. Gauthier, Neutrophil elastase, proteinase 3 , and cathepsin $\mathrm{G}$ as therapeutic targets in human diseases, Pharmacol. Rev. 62 (4) (2010) 726-759.

[84] M. A. Nenke, J. G. Lewis, W. Rankin, D. Shaw, D. J. Torpy, Corticosteroid-binding globulin cleavage may be pathogen-dependent in bloodstream infection, Clin. Chim. Acta 464 (2017) 176-181.

[85] M. A. Nenke, M. Holmes, W. Rankin, J. G. Lewis, D. J. Torpy, Corticosteroid-binding globulin cleavage is paradoxically reduced in alpha-1 antitrypsin deficiency: Implications for cortisol homeostasis, Clin. Chim. Acta 452 (2016) 27-31.

[86] F. Roelfsema, G. Van den Berg, M. Frölich, J. D. Veldhuis, A. Van Eijk, M. Buurman, B. Etman, Sex-dependent alteration in cortisol response to endogenous adrenocorticotropin., J. Clin. Endocrinol. Metab. 77 (1) (1993) 234-240.

[87] Y. Sofer, E. Osher, R. Limor, G. Shefer, Y. Marcus, I. Shapira, K. Tordjman, Y. Greenman, S. Berliner, N. Stern, Gender determines serum free cortisol: higher levels in men , Endocr. Pract. 22 (12) (2016) 1415-1421.

[88] I. Perogamvros, M. Kayahara, P. J. Trainer, D. W. Ray, Serum regulates cortisol bioactivity by corticosteroid-binding globulin-dependent 
and independent mechanisms, as revealed by combined bioassay and physicochemical assay approaches, Clin. Endocrinol. 75 (1) (2011) 3138 .

[89] J. T. Ho, J. G. Lewis, P. O'Loughlin, C. J. Bagley, R. Romero, G. A. Dekker, D. J. Torpy, Reduced maternal corticosteroid-binding globulin and cortisol levels in pre-eclampsia and gamete recipient pregnancies, Clin. Endocrinol. 66 (6) (2007) 869-877.

[90] C. M. Mendel, The free hormone hypothesis: a physiologically based mathematical model, Endocr. Rev. 10 (3) (1989) 232-274.

[91] O. A. Strel'chyonok, G. V. Avvakumov, Interaction of human CBG with cell membranes, J. Steroid Biochem. Mol. Biol. 40 (4) (1991) 795-803.

[92] B. J. Carroll, F. Cassidy, D. Naftolowitz, N. E. Tatham, W. H. Wilson, A. Iranmanesh, P. Y. Liu, J. D. Veldhuis, Pathophysiology of hypercortisolism in depression, Acta Psychiatr. Scand. 115 (2007) 90-103.

[93] J. D. Veldhuis, A. Iranmanesh, G. Lizarralde, M. L. Johnson, Amplitude modulation of a burstlike mode of cortisol secretion subserves the circadian glucocorticoid rhythm, Am. J. Physiol. Endocrinol. Metab. 257 (1) (1989) E6-E14.

[94] F. Vinther, M. Andersen, J. T. Ottesen, The Minimal Model of the Hypothalamic-Pituitary-Adrenal Axis, J. Math. Biol. 63 (4) (2010) 663690.

[95] S. L. Shmakov, A universal method of solving quartic equations, Int. J. Pure Appl. Math. 71 (2) (2011) 251-259.

[96] T. Co, Real Roots of Cubic Equation, URL http://pages.mtu.edu/ rtbco/cm3230/Real_Roots_of_Cubic_Equation.pdf, 2014. 